\title{
Modified Entropy Scaling of the Transport Properties of the Lennard-Jones Fluid
}

\author{
Ian H. Bell, ${ }^{* \dagger}{ }^{\dagger}$ Richard Messerly, ${ }^{\dagger}$ Monika Thol, ${ }^{\ddagger}$ Lorenzo Costigliola, ${ }^{\S}$ and Jeppe C. Dyre ${ }^{\S}$ \\ ${ }^{\dagger}$ Applied Chemicals and Materials Division, National Institute of Standards and Technology, Boulder, Colorado 80305, United \\ States \\ ${ }^{\ddagger}$ Thermodynamics, Ruhr-Universität Bochum, Universitätsstraße 150, 44801 Bochum, Germany \\ ${ }^{\S}$ DNRF Centre “Glass and Time,” IMFUFA, Department of Science and Environment, Roskilde University, Postbox 260, DK-4000 \\ Roskilde, Denmark
}

\section{Supporting Information}

ABSTRACT: Rosenfeld proposed two different scaling approaches to model the transport properties of fluids, separated by 22 years, one valid in the dilute gas, and another in the liquid phase. In this work, we demonstrate that these two limiting cases can be connected through the use of a novel approach to scaling transport properties and a bridging function. This approach, which is empirical and not derived from theory, is used to generate reference correlations for the transport properties of the Lennard-Jones 12-6 fluid of viscosity, thermal conductivity, and self-diffusion. This approach, with a very simple functional form, allows for the reproduction of the most accurate simulation data to within nearly their statistical uncertainty. The correlations are used to confirm that for the Lennard-Jones fluid the appropriately scaled transport properties are nearly monovariate functions of the excess entropy from low-density gases into the supercooled phase and up to extreme temperatures. This study represents the most comprehensive metastudy of the transport properties of the Lennard-Jones fluid to date.

\section{INTRODUCTION}

In 1977 Rosenfeld published a paper suggesting that transport properties are controlled by the excess entropy $s_{\mathrm{ex}}{ }^{1}$ the excess entropy is the change in entropy compared to the ideal gas at the same temperature and density caused by intermolecular interactions. Molecular interactions tend to reduce the entropy compared to that of the noninteracting ideal gas at the same temperature and density; therefore, the excess entropy is generally negative. In other words, interactions between molecules reduce the allowable microstates of the system, resulting in a reduction in entropy (compared to the ideal gas at the same temperature and density).

In this paper we follow the tradition of physical chemistry by defining the excess entropy by

$$
s_{\mathrm{ex}}(T, \rho) \equiv s(T, \rho)-s^{0}(T, \rho)
$$

where $s$ is the total entropy per particle and $s^{0}$ is the ideal gas entropy per particle. The thermodynamic quantity $s_{\mathrm{ex}}$ goes by many other names in the literature, including residual entropy, ${ }^{2-5}$ internal entropy, ${ }^{1}$ and isometric residual entropy. ${ }^{6}$ In the chemical thermodynamics community, the term excess entropy is strictly reserved for deviations from Raoult's law, ${ }^{7}$ and the term residual entropy is used in the place of excess entropy, though the residual entropy has precisely the same

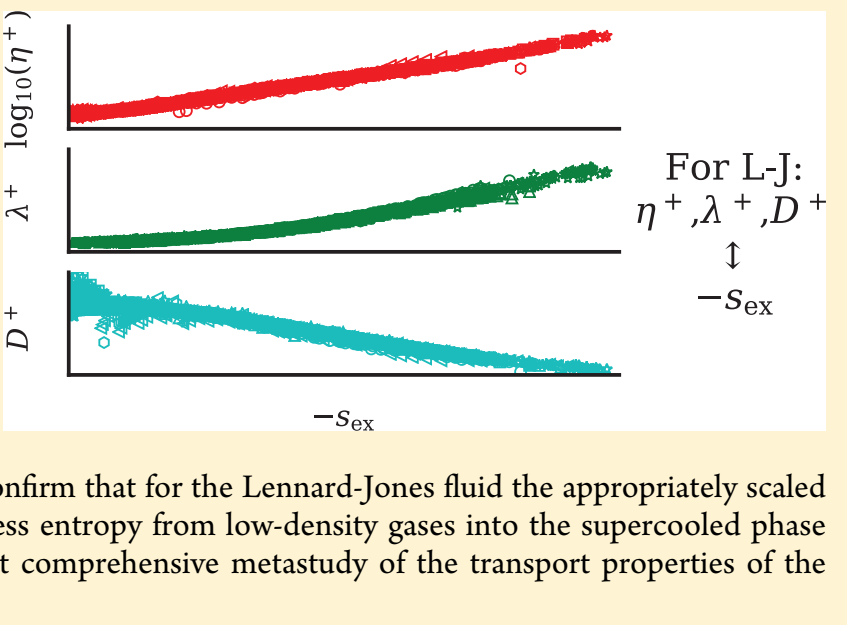

definition as the excess entropy in eq 1 . There are at least two definitions for excess entropy, one based upon the difference in entropy between the given state and the ideal gas at the same temperature and density and another at the same temperature and pressure. Whenever any of these entropy difference terms are used, they should be defined explicitly.

In his seminal work, Rosenfeld considered the available simulations for the Lennard-Jones (LJ) 12-6 fluid, which included four simulations for shear viscosity, and four simulations for the self-diffusion coefficient. Since then, the number of simulations for the Lennard-Jones 12-6 fluid has increased dramatically, due to the popular approach of modeling the thermodynamic and transport properties of real fluids by analogy with the Lennard-Jones 12-6 fluid. ${ }^{2,8}$

Here, in this work, we have collected the most comprehensive set of transport data for the Lennard-Jones 12-6 fluid from the literature and applied a novel scaling approach that is based on approaches proposed by Rosenfeld. ${ }^{1,9}$ Following the approach of ref 2, we use referencequality thermodynamic models in order to minimize the uncertainty in excess entropy.

Received: June 18, 2019

Published: June 26, 2019 
From the analysis of the data collected in this work for the Lennard-Jones fluid, we offer the following new insights:

- Modified entropy scaling allows for a means of connecting the transport physics of the gas phase with that of the liquid phase with a single independent variable, the excess entropy. The macroscopically scaled linear transport coefficients are entirely governed by the excess entropy, and the scaling between excess entropy and scaled transport properties is valid from the dilutegas limit to supercooled states and states at very high temperatures.

- The empirical correlations for the transport coefficients allow for a means of quantifying the monovariability of this scaling, and the deviations of the correlations from the simulation data are shown to be approximately equal to the uncertainty of the simulations.

- The scaled transport data for the Lennard-Jones truncated and shifted (LJTS) and Lennard-Jones truncated with long-range corrections (LJT+LRC) potentials cannot be distinguished in these scaled coordinates. Therefore, impacts from truncation of the potential on the scaled transport property and residual entropy approximately cancel.

- This scaling demonstrates monovariate collapse of the transport data as a function of excess entropy even where isomorph theory should not be valid $(R<0.9)$. Entropy scaling appears to have a wider range of applicability than isomorph theory.

\section{BACKGROUND}

To demonstrate the relationship between excess entropy and transport properties from the numerical data available at the time, Rosenfeld converted the relevant physical properties (diffusion coefficient, viscosity, and thermal conductivity) into dimensionless quantities by using a particular unit system, sometimes referred to as macroscopic reduced units. These units, which were used already in the 1930s by Andrade in his theory of viscosity, ${ }^{10-12}$ are based on the length unit $l_{0}=\rho_{\mathrm{N}}^{-1 / 3}$ in which $\rho_{\mathrm{N}}=N / V$ is the particle number density ( $V$ is volume), the energy unit $e_{0}=k_{\mathrm{B}} T$, and a time unit constructed from $l_{0}$ and $e_{0}$ as follows: $t_{0}=l_{0} / \sqrt{e_{0} / m}$ ( $m$ is the particle mass; $t_{0}$ is the time it takes to travel the distance $l_{0}$ if the particle has thermal velocity). Thus, $t_{0}=\rho_{\mathrm{N}}^{-1 / 3} \sqrt{m / k_{\mathrm{B}} T}$.

For instance, the diffusion coefficient $D$ is made dimensionless by dividing by $l_{0}^{2} / t_{0}$, denoted as the macroscopically reduced diffusion coefficient by $\widetilde{D}$. Thus

$$
\widetilde{D} \equiv \rho_{\mathrm{N}}^{1 / 3} \sqrt{m / k_{\mathrm{B}} T} D
$$

In this section, we henceforth focus on $D$; the viscosity and heat conductivity are treated analogously.

Rosenfeld's excess-entropy scaling is the observation that $\widetilde{D}$ is a unique function of $s_{\mathrm{ex}}$ throughout the thermodynamic phase diagram. For the "ordinary" condensed liquid phase, i.e., not very far from the freezing line, the isomorph theory explains entropy scaling. ${ }^{13}$ In this theoretical framework, which works whenever the equilibrium fluctuations in the virial of the system are strongly correlated with those in the potential energy, ${ }^{14,15}$ excess-entropy scaling is a consequence of "hidden scale invariance". ${ }^{13,16}$ This is the condition such that the ordering of potential energies of system configurations is maintained upon a uniform scaling of all particle coordinates (keeping all intramolecular distances unchanged). ${ }^{13,17,18}$ Hidden scale invariance applies to a good approximation for the condensed liquid phase (as well as the crystalline phase) of most or all metals and van der Waals bonded systems, whereas it is not expected to work for systems with strong directional bonds like hydrogen-bonded or covalently bonded systems. Ionic liquids and dipolar systems constitute an in-between case for which hidden scale invariance is expected to apply if the interactions are not very strong.

Hidden scale invariance has been demonstrated in extensive computer simulations, as well as in experiments, on van der Waals bonded molecular liquids (see, e.g., the reviews given in refs 13,16 , and 18). In particular, the LJ system has hidden scale invariance in the condensed-phase part of the phase diagram. However, the virial potential-energy correlations of the LJ system decrease when the gas phase is approached (see Section 4.1). Interestingly, excess-entropy scaling still works in this part of the phase diagram. In fact, Rosenfeld in 1999 showed that the dilute-gas phase obeys excess-entropy scaling. ${ }^{9}$ How does one rationalize this fact within a theoretical framework that generalizes the isomorph theory? This is not clear, but we have identified a novel way of scaling that addresses the challenge of extending excess-entropy scaling to the gas phase ${ }^{2}$ and which may provide a good starting point for further theoretical developments.

In order to justify the new modified excess-entropy scaling, which was introduced as a practical tool in ref 2 , we first consider the liquid phase. The physics of hidden scale invariance is the nontrivial fact that the relevant length scale is not the length parameter $\sigma$ of the LJ pair potential. This scale is relevant for determining the density at moderate pressures, but hidden scale invariance is independent of the pressure. $^{15,16,18,19}$ Instead, the relevant length scale is the average interparticle distance, which is $\rho_{\mathrm{N}}^{-1 / 3}$.

Things are different in the gas phase. Here, the molecule size, which can be identified with the $\sigma$ of the LJ pair potential, is one relevant length scale. There is, however, one more relevant parameter, namely, the so-called mean-free path $l$ (the average distance traveled between collisions). In the gas phase the relation between the three lengths, where the third length is the average particle distance determined by $\rho_{\mathrm{N}}$, is given by ${ }^{20}$

$$
\sigma^{2} l \rho_{\mathrm{N}} \sim 1
$$

The diffusion constant scales as follows ${ }^{20}$

$$
D \sim l v
$$

in which $v \sim \sqrt{k_{\mathrm{B}} T / m}$ is the thermal velocity. These two simple equations are the most important identities of kinetic theory, and they are direct consequences of the physical picture that a gas behaves like a collection of continuously colliding hard spheres. In eq 3 , as number density $\rho_{\mathrm{N}}$ goes to zero, $l$ goes to $\infty$, and as a consequence, the self-diffusion from eq 4 diverges for any given temperature. We proceed to derive Rosenfeld's 1999 result that excess-entropy scaling works even in the gas phase, with the following prediction:

$$
\widetilde{D} \propto\left(-s_{\text {ex }} / k_{\mathrm{B}}\right)^{-2 / 3}\left(\rho_{\mathrm{N}} \rightarrow 0\right)
$$

Combining eq 3 with eq 4 we get

$$
D \sim \frac{v}{\sigma^{2} \rho_{\mathrm{N}}}
$$

which via eq 2 implies that 


$$
\widetilde{D} \sim \frac{\rho_{\mathrm{N}}^{1 / 3}}{\sigma^{2} \rho_{\mathrm{N}}}=\left(\rho_{\mathrm{N}} \sigma^{3}\right)^{-2 / 3}
$$

On the other hand, $s_{\mathrm{ex}} \rightarrow 0$ as density goes to zero, so a firstorder Taylor expansion results in $s_{\mathrm{ex}} / k_{\mathrm{B}} \sim-\rho_{\mathrm{N}} \sigma^{3}$ at low densities $\left(s_{\mathrm{ex}} / k_{\mathrm{B}}\right.$ is dimensionless and can only depend on density via the dimensionless combination $\rho_{\mathrm{N}} \sigma^{3}$; recall also that $s_{\mathrm{ex}}$ is negative). Inserting this into eq 7 leads to the following low-density scaling

$$
\widetilde{D} \sim\left(-s_{\mathrm{ex}} / k_{\mathrm{B}}\right)^{-2 / 3}
$$

Note that this applies even if the effective hard-sphere radius $\sigma$ depends both on temperature and density.

How does one smoothly connect the gas- and liquid-phase descriptions? The challenge is that while the only relevant length scale in the liquid phase is the average interparticle distance $l_{0} \equiv \rho_{\mathrm{N}}^{-1 / 3}$, the gas phase has two relevant length scales, $l$ and $\sigma$, connected by eq 3 . This suggests defining different reduced units for the gas phase in order to arrive at the relevant dimensionless variables like $\widetilde{D}$. On the other hand, the macroscopic reduced units of the traditional Rosenfeld scaling have the advantage that no knowledge about the molecular size is required; i.e., these are pragmatic units that directly refer to experiments.

Instead of changing the unit system, we propose to keep Rosenfeld's reduced units throughout the phase diagram but make an entropy-dependent correction that removes the gasphase divergence of eq 8 . Thus, we define the following new reduced variables:

$$
D^{+} \equiv\left(-s_{\text {ex }} / k_{\mathrm{B}}\right)^{2 / 3} \widetilde{D}
$$

This is to be used throughout the phase diagram, i.e., also in the liquid phase. Here, however, the new factor $\left(-s_{\mathrm{ex}} / k_{\mathrm{B}}\right)^{2 / 3}$ introduces an excess-entropy dependence that is approximately exponential throughout the liquid phase and when approaching the melting line (and continuing into the supercooled phase if that is relevant).

2.1. Practical Applications. In recent years there has been a growing interest in making use of Rosenfeld's discoveries to develop empirical correlations of the transport properties of real fluids over the entire fluid domain. The review of Dyre ${ }^{13}$ provides a comprehensive view of the application of excessentropy scaling to the transport properties of real fluids, and we discuss a few studies relevant to this work here. One of the bedeviling features of Rosenfeld's entropy scaling is that the scaled properties all diverge at zero density (zero excess entropy), which has led to a number of approaches for means of circumventing this problem.

One approach for managing the zero-density divergence is to subtract the dilute-gas transport properties from the value of the dense phase, but not apply macroscopic scaling. ${ }^{21}$ This approach yields the correct values in the zero-density limit but does not utilize the macroscopic scaling required for the application of isomorph theory.

Another approach proposed in the literature is to divide the transport properties by their value in the dilute-gas limit. $3,22-28$ While these formulations are able to quite accurately empirically fit the transport properties of real fluids, they are not suitable to model small and spherically symmetric particles such as the Lennard-Jones fluid. ${ }^{2}$ The weakness of the zerodensity-limit-scaling approach is that the zero-density-limitscaled transport properties are not equivalent to macroscopic reduction of the transport properties, a necessary condition for the application of isomorph theory.

The group of Truskett proposed the generalized Rosenfeld scaling ${ }^{29-31}$ which shares features with the scaling employed in this work. The second virial coefficient term $B_{2}+T \mathrm{~d} B_{2} / \mathrm{d} T$ is used to scale the self-diffusion in the dilute gas, which is quite similar to the term $\left(B_{2}+T \mathrm{~d} B_{2} / \mathrm{d} T\right)^{2 / 3}$ used in this work at low densities.

The scaling approach proposed in this work has a wellcharacterized limit at zero density, does not have the zerodensity divergence of Rosenfeld's liquid-phase scaling, and retains the required macroscopic scaling throughout the dense phases. As such, we believe this scaling repairs the deficiencies of prior scaling approaches based on Rosenfeld's proposals and allows for the application of the same scaling approach for all fluid states.

\section{POTENTIALS}

The Lennard-Jones $12-6$ potential is the canonical pair potential for a spherically symmetric monatomic molecule (e.g., the noble gases) and, as such, has been studied extensively. This potential is given by

$$
V_{\mathrm{LJ}}(r)=4 \varepsilon\left(\left(\frac{\sigma}{r}\right)^{12}-\left(\frac{\sigma}{r}\right)^{6}\right)
$$

Two common methods exist for simulating the LennardJones potential in a finite box with periodic boundary conditions. The first approach is to truncate the potential $\left(V_{\mathrm{LJT}}\right)$ at a cutoff distance $\left(r_{\text {cut }}\right)$ :

$$
V_{\mathrm{LJT}}(r)= \begin{cases}V_{\mathrm{LJ}}(r) & r<r_{\text {cut }} \\ 0 & r \geq r_{\text {cut }}\end{cases}
$$

Analytical long-range corrections (LRC) to the energy and pressure are often used to account for neglecting interactions at $r>r_{\text {cut }}$. The second approach is to truncate and shift the potential $\left(V_{\mathrm{LJTS}}\right)$, yielding

$$
V_{\mathrm{LJTS}}(r)= \begin{cases}V_{\mathrm{LJ}}(r)-V_{\mathrm{LJ}}\left(r_{\text {cut }}\right) & r<r_{\text {cut }} \\ 0 & r \geq r_{\text {cut }}\end{cases}
$$

For both approaches, frequently $r_{\text {cut }}=2.5 \sigma$.

The Lennard-Jones 12-6 potential has the characteristics of a real molecular fluid with both repulsion and attraction; it has liquid phases, a vapor-liquid critical point, and vapor-liquid phase equilibrium. The family of fully repulsive potentials include the inverse-power-law, the repulsive part of the Weeks-Chandler-Anderson decomposition of the LennardJones potential, and many others like the exponentially repulsive EXP pair potential. ${ }^{32,33}$ The fully repulsive potentials do not show these liquid-like features; they have only a fluid phase and a solid phase.

The exponent 6 of the attractive part of the Lennard-Jones 12-6 potential is consistent with theory, ${ }^{34,35}$ while the exponent 12 of the repulsive part has no rigorous justification. Generalizing this repulsive exponent yields the Mie family of Lennard-Jones potentials, which have also been considered as model systems for transport property modeling. ${ }^{36,37}$ Even though the exponent 12 cannot be justified theoretically, it has been shown to be suitable to model both thermodynamics and transport properties. ${ }^{38}$ The equation of state (EOS) of Lafitte et al. ${ }^{39}$ could be used to calculate the excess entropy for the 
Mie potential, but this EOS provides erroneous predictions of some thermodynamic properties.

The inverse-power-law potential (IPL) is directly related to the family of Mie potentials. The functional form of the IPL is given by

$$
V(r)=\varepsilon\left(\frac{\sigma}{r}\right)^{n}
$$

The IPL is sometimes referred to as the soft-sphere potential (usually with $n=12$ ). The IPL potential is a mathematically simple potential; a consequence of its simplicity is that closedform solutions are possible for some of its properties. We will refer to the IPL potentials in the discussion of zero-density limit transport properties in Section 8. Note that excessentropy scaling is exact for all IPL systems.

\section{CLASSICAL ROSENFELD SCALING}

One of the key insights of Rosenfeld is that the macroscopically reduced transport properties are what count. ${ }^{1,9}$ Therefore, the macroscopically reduced transport properties are indicated with a tilde

$$
\begin{aligned}
& \tilde{\lambda}=\frac{\lambda}{k_{\mathrm{B}} \rho_{\mathrm{N}}^{2 / 3} \sqrt{k_{\mathrm{B}} T / m}} \\
& \tilde{\eta}=\frac{\eta}{\rho_{\mathrm{N}}^{2 / 3} \sqrt{m k_{\mathrm{B}} T}} \\
& \widetilde{D}=\frac{\rho_{\mathrm{N}}^{1 / 3} D}{\sqrt{k_{\mathrm{B}} T / m}}
\end{aligned}
$$

where $\lambda$ is the thermal conductivity, $\eta$ is the viscosity, $D$ is the self-diffusion coefficient, $m$ is the mass of one particle, $k_{\mathrm{B}}$ is Boltzmann's constant $1.380649 \times 10^{-23} \mathrm{~J} \mathrm{~K}^{-1}, 40$ and $T$ is the temperature.

In molecular simulations, reduced simulation units are used, which results in the identical definitions for the macroscopically reduced transport properties in simulation units given by

$$
\begin{aligned}
& \tilde{\lambda}=\frac{\lambda^{*}}{\left(\rho^{*}\right)^{2 / 3} \sqrt{T^{*}}} \\
& \tilde{\eta}=\frac{\eta^{*}}{\left(\rho^{*}\right)^{2 / 3} \sqrt{T^{*}}} \\
& \tilde{D}=\frac{\left(\rho^{*}\right)^{1 / 3} D^{*}}{\sqrt{T^{*}}}=\frac{\left(\rho^{*} D^{*}\right)}{\left(\rho^{*}\right)^{2 / 3} \sqrt{T^{*}}}
\end{aligned}
$$

where $\lambda^{*}=\lambda \sigma^{2} /\left(k_{\mathrm{B}} \sqrt{\varepsilon / m}\right), D^{*}=D /(\sigma \sqrt{\varepsilon / m}), \eta^{*}=\eta \sigma^{2} / \sqrt{m \varepsilon}$, $\rho^{*}=\rho_{\mathrm{N}} \sigma^{3}, T^{*}=k_{\mathrm{B}} T / \varepsilon$, and $\rho^{*} D^{*}=\left(\rho_{\mathrm{N}} D\right)\left(\sigma^{2} / \sqrt{\varepsilon / m}\right)$. In the case of self-diffusion, the second suggestive form highlights the similarity of the reduced transport properties in simulation units; each is a reduced transport property divided by $\left(\rho^{*}\right)^{2 / 3} \sqrt{T^{*}}$. The value $\rho^{*} D^{*}$ is employed as the scaled transport property rather than $D^{*}$ because while $D^{*}$ diverges in the zero-density limit, $\rho^{*} D^{*}$ remains finite. ${ }^{41}$

Before describing in depth the considered molecular simulations and their methodology, we first introduce the Rosenfeld-scaled transport properties for the Lennard-Jones fluid. The set of data obtained was originally based on the collection of Lautenschlaeger ${ }^{42}$ with extensive data corrections and the addition of data sets from other publications. Figures 1-3 present the macroscopically scaled transport properties as

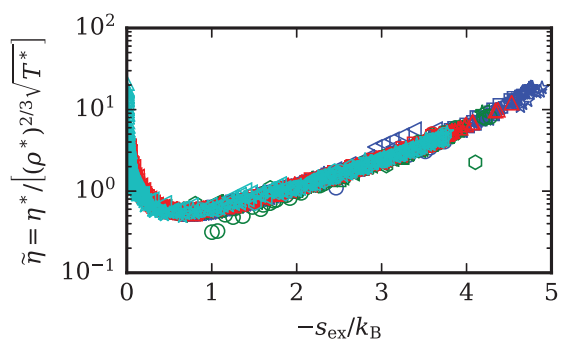

Figure 1. Rosenfeld-scaled viscosity values from simulations ${ }^{42,42-62}$ for the Lennard-Jones 12-6 potential. Each marker/color pair is associated with a given data set. A larger version of this figure is available in the SI (Figure S1), along with a legend describing each data set.

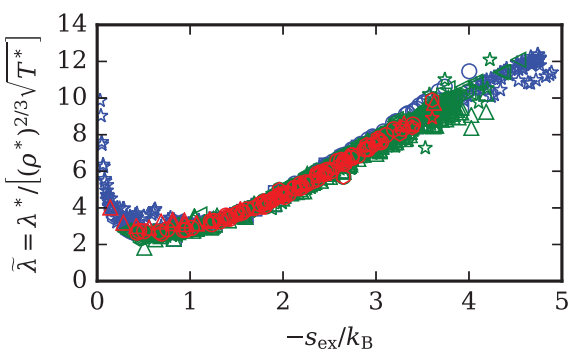

Figure 2. Rosenfeld-scaled thermal conductivity values from simulations ${ }^{42,42,46,50,52-55,57,62-68}$ for the Lennard-Jones $12-6$ potential. Each marker/color pair is associated with a given data set. A larger version of this figure is available in the SI (Figure S2), along with a legend describing each data set.

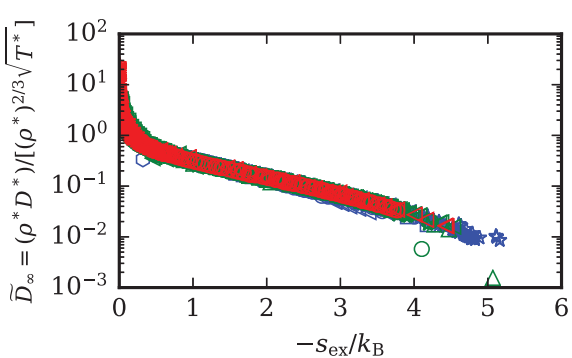

Figure 3. Rosenfeld-scaled self-diffusion values from simulations ${ }^{41,42,42,46-48,50-53,58,59,69-71}$ for the Lennard-Jones 12-6 potential. Each marker/color pair is associated with a given data set. A larger version of this figure is available in the SI (Figure S3), along with a legend describing each data set.

a function of the excess entropy. The evaluation of the excess entropy is explained in Section 6.1. This scaling results in a divergence of all three properties at zero density (zero excess entropy) due to the presence of the term $1 /\left(\rho^{*}\right)^{2 / 3}$. The novel scaling proposed in this work does not have the zero-densitylimit divergence.

4.1. Range of Applicability. According to the isomorph theory, ${ }^{15,32,72-76}$ when the correlation between the virial and the potential energy is strong, the macroscopically reduced transport properties according to eqs 17-19 should be monovariate functions of the excess entropy. It is common practice within the isomorph literature ${ }^{14,77}$ to define a correlation coefficient $R$ greater than 0.9 as being "strongly correlating". 
The correlation coefficient $R$ between the virial $W$ and potential energy $U$ fluctuations can be calculated from NVT simulations using the formula

$$
R\left(T^{*}, \rho^{*}\right)=\frac{\langle\Delta W \Delta U\rangle}{\sqrt{\left\langle(\Delta W)^{2}\right\rangle\left\langle(\Delta U)^{2}\right\rangle}}
$$

where $\Delta$ denotes instantaneous equilibrium fluctuations from the mean value and the brackets that an NVT ensemble average is taken. In the case of the LJ system the correlation coefficient $R$ is increasing toward its maximum value 1 when either density or temperature is increased, as can be seen from Figure 4. State points which are close to the LJ freezing

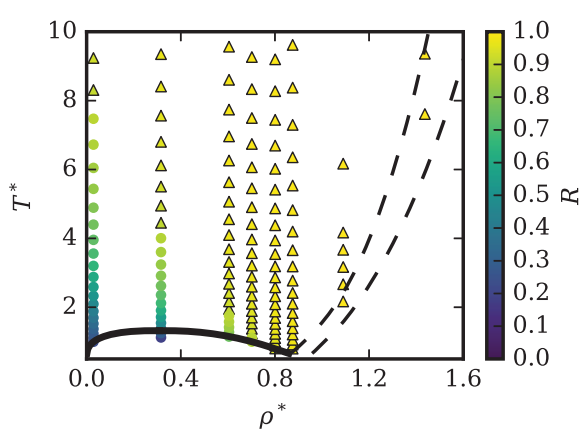

Figure 4. Correlation coefficient $R$ for the Lennard-Jones $12-6$ potential from the simulations of ref 47 (for $\rho^{*} \geq 0.605$ ) and simulations from this work (for $\rho^{*}<0.605$ ). Triangles indicate state points with $R>0.9$, and circles indicate state points with $R<0.9$. The solidus and liquidus lines (indicated by dashed lines) were obtained from ref 78 and from refs 79 and 80 , respectively. The thick solid line is the vapor-liquid equilibrium for the full $\mathrm{LJ}$ potential.

(liquidus) line have a high correlation coefficient $(R>0.9)$ while approaching the gas-liquid coexistence leads to a decrement in $R$.

\section{LITERATURE REVIEW}

We will work with the simulation data presented in the literature. An overview of the simulation results that are available is presented in graphical form in Figure 5. Tables S1-
S3 in the SI describe the simulation setup for each of the literature studies. In general, these various methods and setup parameters yield consistent transport property estimates. However, the simulation specifications are important to consider when elucidating the possible source for a discrepancy between two data sets and for ensuring reproducibility of results. A brief description of the various simulation approaches follows.

5.1. Simulation Method. Several methods exist for computing transport properties with molecular simulation. These methods are classified as either equilibrium or nonequilibrium molecular dynamics. Equilibrium molecular dynamics (EMD) follows an unperturbed MD trajectory, whereas nonequilibrium molecular dynamics (NEMD) perturbs the system and observes the response to this perturbation. $^{81,82}$

EMD is ideal for computing multiple transport properties $(D, \eta$, and $\lambda)$ from a single simulation. ${ }^{83}$ By contrast, traditional NEMD methods are only capable of predicting a single property from a given NEMD simulation, and typically, a sequence of NEMD simulations is required to extrapolate to the zero perturbation limit. The exception is the recently proposed NEMD approach utilized by Lautenschlaeger et al., which yields all three transport properties from a single simulation. $^{84}$

Two related EMD methods are available for computing transport properties, namely, the Green-Kubo (GK) method and the Einstein relationship (ER). In principle, these two postprocessing approaches should yield indistinguishable results; however, due to numerical approximations and some user judgment, deviations of a few percent between GK and ER are not uncommon. ${ }^{83,85}$

Several NEMD methods exist where different perturbations are applied to the system. ${ }^{82}$ We refer the reader to the literature for details regarding these various NEMD methods. $^{49,84,86-91}$

For the literature studies considered in this work, all selfdiffusivity data (with the exception of those from Lautenschlaeger et al.) were obtained with EMD, while EMD and NEMD were equally popular for computing thermal

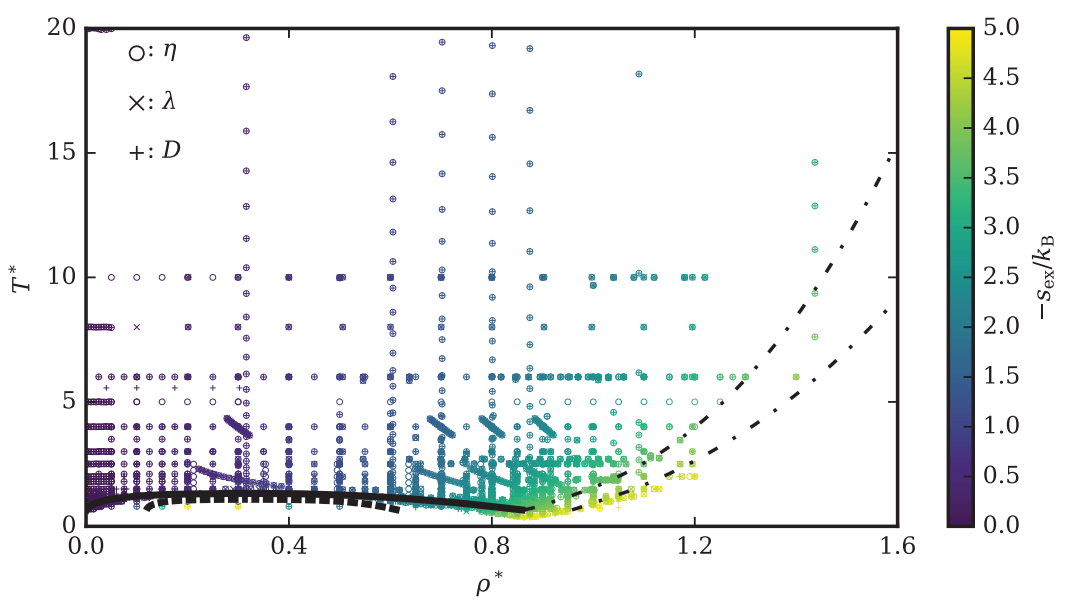

Figure 5. Distribution of transport property simulations in the phase diagram as well as excess entropy calculated for each point. The graphic is truncated at $T^{*}=20$ (data are available up to $\left.T^{*}=10800\right)$ and at $\rho^{*}=1.6$ (data are available up to $\rho^{*}=3.39$ ). The dashed-dotted lines correspond to the solidus and liquidus lines and were obtained from ref 78 and refs 79 and 80 , respectively. The excess entropy $-s^{\mathrm{r}} / k_{\mathrm{B}}$ varies from 3.6 to 3.9 along the liquidus line for $T^{*}<15$, and from 4.3 to 4.7 for the solidus line for $T^{*}<15$. The thick solid line is the vapor-liquid equilibrium for the full LJ potential, and the thick dashed line is the vapor-liquid equilibrium for the LJTS potential. 
conductivity and viscosity. Frequently, NEMD values are verified by comparison with their simpler EMD counterparts, such that some studies report both EMD and NEMD results. Agreement between EMD and NEMD values is typically within the combined uncertainties. ${ }^{92}$

GK is used for computing thermal conductivity and viscosity in nearly all of the EMD studies, while GK and ER are equally popular for computing self-diffusivity. No single NEMD method was found to be more popular than the others.

5.2. Simulation Ensemble. Another important distinction among the literature EMD simulations is the use of the microcanonical (NVE) or canonical (NVT) ensembles. Both the NVE and NVT ensembles utilize a constant number of molecules $(N)$ and volume $(V)$. The difference is that the NVE ensemble enforces a constant overall energy, while the NVT ensemble maintains a constant temperature (or kinetic energy) by applying a thermostat. ${ }^{93-97}$ If implemented properly, both the NVE and NVT ensembles are considered reliable for estimating transport properties, although advantages and disadvantages exist for each ensemble. ${ }^{98}$

For example, one advantage of the NVT ensemble is that transport properties are generally desired at a given temperature, not a given energy. One disadvantage of the NVT ensemble is the potential effect of certain thermostats and/or thermostat coupling strengths on the resultant system dynamics, whereas NVE dynamics are undisturbed by these artificial thermostats. ${ }^{98}$

Approximately the same number of EMD studies utilize the NVE or NVT ensemble. Including the NEMD simulations that also utilize thermostats, four different thermostats are found in the literature with similar popularity.

5.3. Finite-Size Effects. 5.3.1. Number of Molecules. Because the literature spans approximately 40 years and computational speed has increased dramatically during that time, the number of molecules simulated in recent studies is often an order of magnitude greater than those in earlier studies. The degree to which results are impacted by the number of simulation particles $N$ depends on both the transport property and the state point. While a well-known linear relationship exists to relate the system size $\left(1 / N^{1 / 3}\right)$ to $D$ (see below), ${ }^{99,100}$ the relationship is less-understood for $\lambda$ and $\eta$. Fortunately, the influence of $N$ on $\eta$ and $\lambda$ is typically negligible compared to that for $D$.

In the case of self-diffusion, the analytic infinite-particlecount self-diffusion limit $D_{\infty}$ can be obtained by one of two methods:

1. Multiple simulation runs are performed for several numbers of particles $N$. The infinite-particle-count selfdiffusion is obtained by extrapolating the linear curve fit of $D$ versus $1 / N^{1 / 3}$ to $\left(1 / N^{1 / 3}=0\right)$. This has the significant disadvantage that multiple simulations must be done at every state point.

2. Apply the empirical finite-size correction term given by ref 99

$$
D_{\infty}=D_{N}+\frac{k_{\mathrm{B}} T \mathcal{X}}{6 \pi \eta L}
$$

where $L=\left(N / \rho_{\mathrm{N}}\right)^{1 / 3}$, and $\mathcal{X}=2.837298$. Conversion to simulation units yields

$$
D_{\infty}^{*}=D_{\mathrm{N}}^{*}+\frac{T^{*} \mathcal{X}}{6 \pi \eta^{*} L^{*}}
$$

where $L^{*}=\left(N / \rho^{*}\right)^{1 / 3}$.

In this work the second method was selected; the correction term was applied to every self-diffusion data point. Unfortunately, the method of ref 99 was originally validated for a rather narrow range of state points.

The viscosity $\eta^{*}$ used in eq 22 is evaluated from the correlation developed below in eq 54. This method has the disadvantage that the uncertainty of the viscosity correlation will "infect" the self-diffusion data corrected for finite-size effects. But this is a more satisfactory situation than the converse: errors in self-diffusion of more than $20 \%$ are caused by small particle counts when the correction for finite-size effects is ignored (see SI Appendix, Figure S8). The inclusion of the uncertainty in the viscosity correlation increases the uncertainty in the self-diffusion. For instance, from an uncertainty propagation analysis (see the SI, Section 3.3), considering worse-case uncertainty of the viscosity correlation of $10 \%$, the uncertainty in the self-diffusion of Meier ${ }^{41}$ increases from an uncertainty of roughly $1 \%$ to an uncertainty of never more than $1.3 \%$ for the simulations with 1372 particles; the relative uncertainty for the simulations of Meier with fewer particles are greater, but still never more than $1.7 \%$.

5.3.2. Cut-Off Distance. For a truncated Lennard-Jones (but not shifted, LJT or LJT+LRC) potential, it was traditionally assumed that simulation results do not depend strongly on the reduced cutoff distance $\left(r_{\text {cut }}^{*}=r_{\text {cut }} / \sigma\right)$ for $r_{\text {cut }}^{*} \geq 2.5$. Some of the more recent studies have utilized significantly larger cutoff distances $\left(r_{\text {cut }}^{*} \geq 5\right)$ to test this assumption. Analytical longrange tail corrections do not impact self-diffusion calculations in the NVE or NVT ensemble, but neglecting this correction may result in deviations for viscosity and thermal conductivity, particularly for shorter values of $r_{\text {cut }}^{*}$. Unfortunately, most studies do not explicitly state whether or not tail corrections are included; i.e., it is not always clear if simulations were performed with the LJT or the LJT+LRC potential. A few studies also utilize a Lennard-Jones truncated-and-shifted (LJTS) potential with $r_{\text {cut }}^{*}=2.5$. Although Lautenschlaeger et al. suggest that the LJTS and LJT+LRC potentials yield indistinguishable transport properties at a given $\left(T^{*}, \rho^{*}\right)$ state point, ${ }^{42}$ the equations of state and, thereby, the excess-entropy estimates for the same $T^{*}-\rho^{*}$ state point are different for the LJTS and LJT+LRC potentials.

5.4. Simulation Time. Simulations are typically divided into an equilibration and production stage, where averages are only computed during production. With advancements in computational speed and resources over the past few decades, the reduced simulation times $\left(t^{*}=t /(\sigma \sqrt{m / \varepsilon})\right)$ for both equilibration $\left(t_{\mathrm{eq}}^{*}\right)$ and production $\left(t_{\text {prod }}^{*}\right)$ have increased by a few orders of magnitude. The consequence of the lower $t_{\text {eq }}^{*}$ values prevalent in earlier studies is the risk of poor equilibration, while the lower $t_{\text {prod }}^{*}$ values lead to higher uncertainties from less sampling.

The reduced simulation time-step $\left(\delta t^{*}\right)$ varies by a factor of 5 among studies. Higher values of $\delta t^{*}$ can result in spurious simulation output. ${ }^{101}$ This is particularly problematic at higher temperatures because particles travel at increased velocities, and thus, some configurations may contain extremely closerange (repulsive) interactions. Although lower values of $\delta t^{*}$ are more reliable, they are less preferred in practice because lower $\delta t^{*}$ values increase the elapsed real time to simulate a fixed value of $t_{\text {prod }}^{*}$ 
5.5. Data Curation. A screening was used to separate each data set into primary and secondary categories. The primary data sets, indicated in the sections that follow, were identified on the basis of their coverage of the phase space, their internal consistency, the description of their methodology, and agreement with other data sets.

In addition to categorization of the data sets, we endeavored to identify points that seemed to be typographical errors, for instance, two state points at very similar temperatures and densities with very different transport properties, or data points that did not follow the trends of the neighboring points. Points that were inside the spinodal of the equation of state were not considered because empirical equations of state may yield erroneous values for thermodynamic properties for the thermodynamically unstable states between the spinodals (see for instance Figure S11 in the SI of ref 2). Data points for temperatures $T^{*}>400$ were also removed from further consideration because the collision integral correlations used for the zero-density-limit demonstrate poor high-temperature extrapolation (see the SI, Figure S11a,b).

Inspired by the work of Lautenschlaeger, ${ }^{42}$ we have provided all of the literature data that we collected in comma-separatedvalue tables in the SI; further information is given in the SI. The "FLAG" column in the data collection indicates the reason that we removed the data point, and the presence of an entry in this column causes the data processing script to ignore the given data point. If the column is empty, the point was considered in our analysis.

\section{THERMODYNAMIC MODELS}

The equation of state for any fluid at low and moderate densities can be written in virial expansion form as

$$
Z \equiv \frac{p}{\rho_{\mathrm{N}} k_{\mathrm{B}} T}=1+\sum_{j=2}^{\infty} B_{j}(T) \rho_{\mathrm{N}}^{j-1}
$$

where $Z$ is the compressibility factor, $p$ is the pressure, $\rho_{\mathrm{N}}$ is the number density in particles per volume, $k_{\mathrm{B}}$ is Boltzmann's constant, $T$ is the temperature, and $B_{j}$ is the $j$ th virial coefficient.

For all thermodynamic states, the reduced residual Helmholtz energy $\alpha^{\mathrm{r}}=a^{\mathrm{r}} /\left(k_{\mathrm{B}} T\right)$, where $a^{\mathrm{r}}$ is the residual Helmholtz energy per particle, can be obtained from ${ }^{103}$

$$
\alpha^{\mathrm{r}}=\int_{0}^{\rho_{\mathrm{N}}} \frac{Z\left(T, \rho_{\mathrm{N}}\right)-1}{\rho_{\mathrm{N}}} \mathrm{d} \rho_{\mathrm{N}}
$$

and the negative of the reduced residual entropy can be obtained from

$$
-s^{\mathrm{r}} / k_{\mathrm{B}}=\frac{1}{k_{\mathrm{B}}}\left(\frac{\partial a^{\mathrm{r}}}{\partial T}\right)_{\rho_{\mathrm{N}}}=T\left(\frac{\partial \alpha^{\mathrm{r}}}{\partial T}\right)_{\rho_{\mathrm{N}}}+\alpha^{\mathrm{r}}
$$

where $s^{r}$ is the residual entropy per particle. As a reminder, our definition of the excess entropy is that it is the difference between the total entropy and that of the ideal gas at the same temperature and density, defined to be equal to the residual entropy, and equal to

$$
s_{\text {ex }}(T, \rho) \equiv s^{r}(T, \rho)=s(T, \rho)-s^{0}(T, \rho)
$$

where $s$ is the total entropy per particle and $s^{0}$ is the ideal gas entropy per particle.
In general, if the virial expansion from eq 23 is truncated at $B_{2}\left(B_{j} \equiv 0\right.$ for $\left.j>2\right)$, then the excess entropy (equal to residual entropy) can be given by

$$
-s_{\mathrm{ex}} / k_{\mathrm{B}}=\rho_{\mathrm{N}}\left(T\left(\frac{\mathrm{d} B_{2}}{\mathrm{~d} T}\right)+B_{2}\right)
$$

This result was also used by Krekelberg et al. ${ }^{29}$ in their study of self-diffusion.

6.1. Empirical EOS. Over the past 70 years, the LennardJones potential has been comprehensively investigated by molecular simulation in the entire fluid domain as well as in the solid state. These data were used to set up equations of state by several different authors. An overview of available thermodynamic properties as well as the most prominent equations of state for the fluid region is given by Thol et al. ${ }^{104}$ The most recent fundamental equation of state for the calculation of thermodynamic properties of the LJT+LRC potential was published in the same paper. It is formulated in terms of the Helmholtz energy $a$ as a function of temperature $T$ and density $\rho$. For simplicity, the Helmholtz energy and its independent variables are used in a dimensionless form. Furthermore, the reduced Helmholtz energy $\alpha=a /\left(k_{\mathrm{B}} T\right)$ is separated into an ideal part $\alpha^{0}$ and a residual part $\alpha^{\mathrm{r}}$ with independent variables of reciprocal reduced temperature $\tau=$ $T_{\mathrm{c}}^{*} / T^{*}$ and reduced density $\delta=\rho^{*} / \rho_{\mathrm{c}}^{*}$ :

$$
\alpha(\tau, \delta)=\frac{a^{0}(T, \rho)+a^{\mathrm{r}}(T, \rho)}{k_{\mathrm{B}} T}=\alpha^{0}(\tau, \delta)+\alpha^{\mathrm{r}}(\tau, \delta)
$$

The advantage of applying the Helmholtz energy as the fundamental potential is that it can be used to calculate every thermodynamic state property by combining the equation itself and its partial derivatives with respect to the density and reciprocal temperature:

$$
A_{m n}=A_{m n}^{0}+A_{m n}^{\mathrm{r}}=\tau^{m} \delta^{n}\left(\frac{\partial\left(\alpha^{0}+\alpha^{\mathrm{r}}\right)}{\partial \tau^{m} \partial \delta^{n}}\right)
$$

where $m$ is the order of derivative with respect to the reciprocal temperature and $n$ with respect to the density.

For instance, the isobaric heat capacity can be calculated from

$$
c_{p}^{*}=-\left(A_{20}^{0}+A_{20}^{\mathrm{r}}\right)+\frac{\left(1+A_{01}^{\mathrm{r}}-A_{11}^{\mathrm{r}}\right)^{2}}{1+2 A_{01}^{\mathrm{r}}+A_{02}^{\mathrm{r}}}
$$

Further mathematical relations for other thermodynamic properties are listed in Thol et al. ${ }^{104}$ Today, the most accurate equations of state are empirical multiparameter equations of state with 15-40 temperature- and density-dependent polynomial(-like) and exponential terms. The corresponding parameters are commonly adjusted to thermodynamic state properties such as density, speed of sound, vapor-liquidequilibrium data, etc. Due to the large number of adjustable parameters, a comprehensive data set comprising different thermodynamic properties is needed. However, since these properties are always combinations of derivatives of the Helmholtz energy, it is not possible to optimize the fundamental potential of its derivatives individually. This shortcoming was first addressed by Rutkai et al. ${ }^{105}$ who presented a novel approach for the development of Helmholtz energy equations of state. On the basis of the methodology of Lustig, ${ }^{106,107}$ the Helmholtz energy and its partial derivatives 
up to an arbitrary order can be computed from molecular dynamics simulations. This allows for a direct fitting of the equation of state to the results of molecular dynamics simulations. The fundamental equation of state for the LJT +LRC potential proposed by Thol et al. ${ }^{104}$ is the first equation, which was developed on the basis of this new approach. Only simulation data for the Helmholtz derivatives and exact virial coefficients calculated from statistical mechanics were included in the fitting process. Furthermore, modern fitting techniques (see, e.g., Lemmon et al. ${ }^{108}$ ) were employed, which could be used to ensure a correct extrapolation behavior in the lowtemperature limit as well as at high temperatures, pressures, and densities.

There are a number of other empirical equations of state for the LJ fluid of varying quality and accuracy. ${ }^{109-113}$ Thol et al. also developed an empirical multiparameter equation of state for the truncated-and-shifted potential (LJTS, with $r_{\text {cut }}^{*}=$ 2.5). ${ }^{114}$ The LJT+LRC and LJTS potentials have qualitatively similar behavior, and their important temperatures and densities are summarized in Table 1 . While the critical

Table 1. Available Lennard-Jones Helmholtz-EnergyExplicit Equations of State ${ }^{a}$

\begin{tabular}{|c|c|c|c|c|}
\hline potential & $r_{\text {cut }}^{*}$ & $T_{\mathrm{c}}^{*}$ & $\rho_{\mathrm{c}}^{*}$ & $T_{\text {triple }}^{*}$ \\
\hline $\mathrm{LJT}+\mathrm{LRC}^{104}$ & $L^{* / 2}$ & 1.32 & 0.31 & 0.661 \\
\hline LJTS $^{114}$ & 2.5 & 1.086 & 0.319 & $0.64^{b}$ \\
\hline
\end{tabular}

${ }^{a}$ Critical points correspond to the values that were obtained in the course of the development of the equation of state. $L^{*}=L / \sigma$ where $L$ is the box length, i.e., $L=V^{1 / 3}$. ${ }^{b}$ Estimated from corresponding states plus a correction factor.

densities are quite similar, the critical temperatures differ significantly. To our knowledge, no high-accuracy equations of state exist for any other truncations of the Lennard-Jones 12-6 potential.

The equations of state for LJT+LRC (with $r_{\text {cut }}^{*}=L^{*} / 2$, i.e., half the reduced box length) and LJTS (with $r_{\text {cut }}^{*}=2.5$ ) represent two treatments that are commonly employed in molecular simulations. Nonetheless, there are many simulations that applied neither a truncated potential at half the box length (which typically corresponds to $r_{\text {cut }}^{*}>2.5$ ) nor a truncated-and-shifted potential for $r_{\text {cut }}^{*}=2.5$. Therefore, some approach must be applied to the in-between cases. Our approach was to use the full (LJT+LRC) EOS for all simulations, except for cases where the authors explicitly state that they used a truncated-and-shifted potential (LJTS) with $r_{\text {cut }}^{*}=2.5 .^{42,47}$ For other cases, such as those of the LJTS potential but truncated and shifted at $r_{\text {cut }}^{*}=5$, we utilized the equation of state for the full (LJT+LRC) potential.

\section{SCALED TRANSPORT PROPERTIES}

7.1. Novel Scaling. Rosenfeld ${ }^{9}$ showed that the values of $\tilde{\lambda}$, $\tilde{\eta}$, and $\widetilde{D}$ are each proportional to $\left(-s_{\text {ex }} / k_{\mathrm{B}}\right)^{-2 / 3}$ for dilute gases of finite density modeled by inverse-power-law pair potentials for a virial expansion truncated at the second virial coefficient. The constant of proportionality is a closed-form constant that is only a function of the hardness of the IPL potential. The excess entropy of a dilute gas at zero density is equal to zero by definition. See the SI for a complete rederivation of this result (SI Section 5.1) following the proposal of Rosenfeld. An empirical scaling approach based on the same technique (though it was not fully investigated at that time) was providentially used by Bell ${ }^{2}$ to repair the divergence of $\tilde{\eta}$ in the zero-density limit.

For the Lennard-Jones fluid, as will be shown in the next section, the relationship between excess entropy and transport properties in the zero-density limit is not quite as simple as that of IPL potentials. The IPL potential, while being unsuitable to model real fluids with high accuracy, provides glimpses into theory that inspire more complicated modeling efforts.

We propose new scaled variables $\eta^{+}, \lambda^{+}$, and $D^{+}$, with the feature that these scaled variables do not diverge at zero density and reflect the notion of the traditionally scaled transport properties being proportional to the excess entropy to a power of $-2 / 3$ in dilute gases. Thus, the new reduced transport coefficients $\eta^{+}, \lambda^{+}$, and $D^{+}$are given by

$$
\begin{aligned}
\eta^{+} & \equiv \widetilde{\eta}\left(-s_{\mathrm{ex}} / k_{\mathrm{B}}\right)^{2 / 3} \\
\lambda^{+} & \equiv \widetilde{\lambda}\left(-s_{\mathrm{ex}} / k_{\mathrm{B}}\right)^{2 / 3} \\
D^{+} & \equiv \widetilde{D}\left(-s_{\mathrm{ex}} / k_{\mathrm{B}}\right)^{2 / 3}
\end{aligned}
$$

These ansatz formulations have the characteristic that they are still monovariate relationships between the macroscopically reduced transport properties and the excess entropy, while adding additional desired behavior.

The definitions of the above variables make no assumption about the form of the interactions between particles. These are simply conveniently scaled variables that have some elegant mathematical properties and allow for an effective scaling of transport properties in general, as is demonstrated in the sections that follow.

The selection of the exponent $2 / 3$, while accidental in the work of Bell, ${ }^{2}$ can be shown (SI Appendix, Section 1.3) to be the only exponent that results in a nonzero value of the scaled transport properties for the zero-density limit. That is to say, $2 / 3$ is indeed a special exponent, and no other exponent is possible in this scaling framework that simultaneously satisfies the constraints on behavior in the zero-density limit and the dense phase.

Krekelberg et al., ${ }^{29}$ by making the assumption that the product $\rho^{*} D^{*}$ should be constant in the dilute gas of finite density, arrived at a similar scaled self-diffusion coefficient, though different from the one used here.

7.2. Viscosity. Figure 6 presents the newly scaled viscosity $\eta^{+}$in terms of $-s_{\text {ex }} / k_{\mathrm{B}}$ for the Lennard-Jones potentials studied

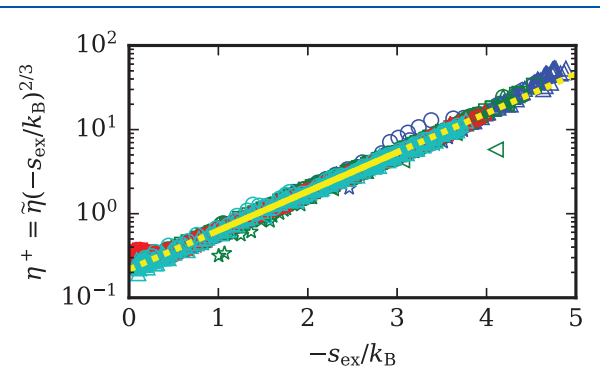

Figure 6. Overview of all of the viscosity data from simulations ${ }^{42,42-62}$ with the novel scaling in eq 31 for the Lennard-Jones 12-6 potential. The solid yellow line represents the correlation from eq 34 , and the dashed lines the extrapolation behavior. Each marker/color pair is associated with a given data set. A larger version of this figure is available in the SI (Figure S4), along with a legend describing each data set. 
here. As can be seen, the collapse of the data for the LennardJones potentials is similarly tight to the "conventional" excessentropy scaling of Rosenfeld in Figure 1. In addition, the relationship between $\eta^{+}$and $-s_{\mathrm{ex}} / k_{\mathrm{B}}$ is approximately exponential (linear in semilogarithmic coordinates) from the dilute gas (at $-s_{\mathrm{ex}} / k_{\mathrm{B}} \approx 0$ ) into the supercooled liquid region, and the divergence at zero density has been removed. As these scaled variables have been proposed for the first time in this work, the theoretical explanation for this quasiexponential behavior remains elusive for now. While a more comprehensive empirical model covering the entire domains of the fluid is presented in Section 9.1, a linear fit to the data in the liquid region for $1 \leq-s_{\mathrm{ex}} / k_{\mathrm{B}} \leq 3$ yields

$$
\eta^{+} \approx 0.2163 \exp \left(1.068\left(-s_{\mathrm{ex}} / k_{\mathrm{B}}\right)\right)
$$

This same quasiexponential behavior no longer holds in the supercooled liquid region (beyond $-s_{\mathrm{ex}} / k_{\mathrm{B}}$ of roughly 3.5), where the data are consistently above the correlation fit to the data for the liquid phase. Similarly, the data in the dilute-gas region at low excess entropy are also consistently above the correlation fit in the liquid region.

Nonetheless, this scaling allows for a coherent picture from zero density into the supercooled liquid, even if additional empiricism might be required to yield a more quantitative agreement between the model and the simulation data.

Figure 7 presents a detailed view of the gaseous region for the Lennard-Jones fluid. The gaseous region, where $-s_{\mathrm{ex}} / k_{\mathrm{B}}$ is

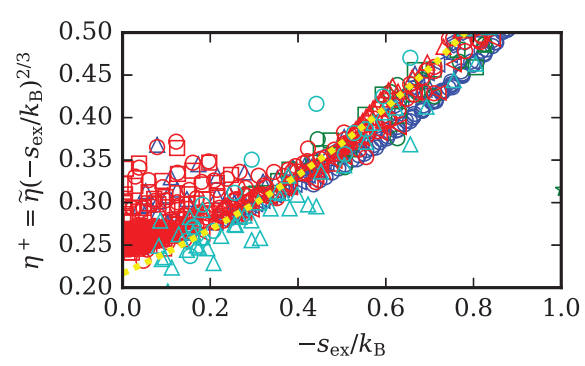

Figure 7. Detailed view of the gaseous region of the novel scaled viscosity data from simulations for the Lennard-Jones 12-6 potential. The dashed yellow line represents the extrapolation of the correlation from eq 34. Each marker/color pair is associated with a given data set. A larger version of this figure is available in the SI (Figure S5), along with a legend describing each data set.

less than approximately 1 , is a region where isomorph theory breaks down (see Figure 4) and the thread linking excess entropy and transport properties should fray. Nevertheless, the dense-phase exponential behavior continues well into the gaseous region. At very low densities (very low $-s_{\mathrm{ex}} / k_{\mathrm{B}}$ ), the dense phase correlation does not reproduce the data even qualitatively, but the fanning-out behavior can be entirely captured (aside from uncertainty of the simulations) by the zero-density limit. When the dilute-gas limiting values are subtracted off (Section 9.1), the dilute-gas viscosity can be reproduced to within its simulation statistical uncertainty. The zero-density limit can be fully described by theory in the case of the Lennard-Jones potential, as shown in Section 8.

7.3. Self-Diffusion. The scaled data for self-diffusion are presented in Figure 8. An absolutely essential element of analyzing the self-diffusion data from the literature is to apply the finite-size correction of Yeh and Hummer ${ }^{99}$ described in Section 5.3.1. Above $-s_{\mathrm{ex}} / k_{\mathrm{B}}$ of approximately 1 , the data tell a

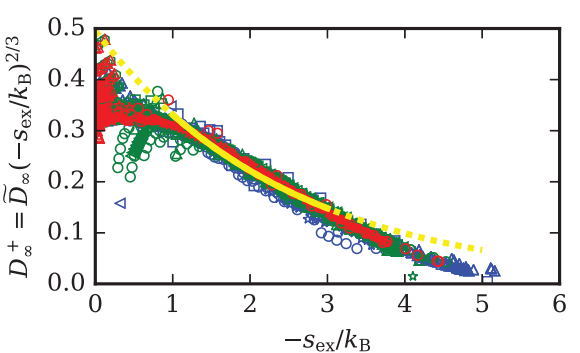

Figure 8. Overview of all of the self-diffusion data from simulations $41,42,42,46-48,50-53,58,59,69-71$ with the novel scaling in eq 33 for the Lennard-Jones 12-6 potential. The yellow curve is the correlation from eq 35. Each marker/color pair is associated with a given data set. A larger version of this figure is available in the SI (Figure S7), along with a legend describing each data set.

very coherent story; the data agree very closely, and the interset deviations are governed by the uncertainty in the viscosity correlation used to correct the self-diffusion data to infinite system size. In concurrence with the viscosity data, the monovariability in the liquid phase continues into the supercooled liquid phase. The zero-density self-diffusion provides a much larger relative contribution to self-diffusion than the equivalent contribution for viscosity. In addition, although we have included all the data in the figure, a number of the gas-phase simulations must be considered as highly suspect. For instance, the self-diffusion data of Lautenschlaeger et al. do not approach the correct zero-density-limit governed by theory and described in the next section (see the SI, Section 3.4.2).

In the same fashion as for viscosity, we fit a simple correlation of the form

$$
D^{+} \approx 0.494 \exp \left(-0.402\left(-s_{\mathrm{ex}} / k_{\mathrm{B}}\right)\right)
$$

proposed by Rosenfeld ${ }^{9}$ to the simulation data for $1 \leq-s_{\mathrm{ex}} / k_{\mathrm{B}}$ $\leq 3$ and, also, show the extrapolation behavior of this curve outside of this region.

In the gaseous region, the data of Meier et al. ${ }^{41}$ are the most comprehensive in their coverage of the thermodynamic phase diagram and also appear to be of the highest quality.

7.4. Thermal Conductivity. Figure 9 shows the scaled thermal conductivity data from molecular dynamics simulations. In the same fashion as for viscosity, we fit a simple correlation of the form

$$
\lambda^{+} \approx 1.377 \exp \left(0.839\left(-s_{\mathrm{ex}} / k_{\mathrm{B}}\right)\right)
$$

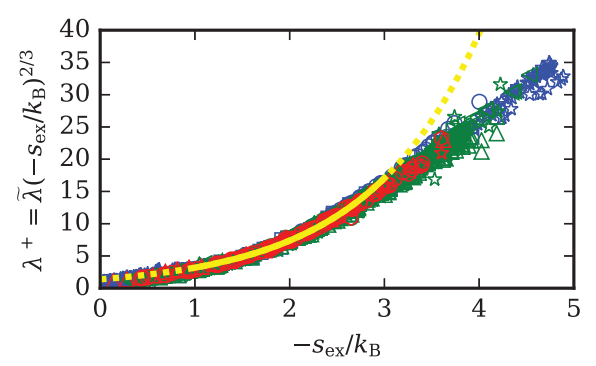

Figure 9. Overview of all the novel scaled thermal conductivity data from simulation ${ }^{42,42,46,50,52-55,57,62-68}$ with the novel scaling in eq 32 for the Lennard-Jones 12-6 potential. The yellow curve is the fitted correlation from eq 36. Each marker/color pair is associated with a given data set. A larger version of this figure is available in the SI (Figure S6), along with a legend describing each data set. 
proposed by Rosenfeld ${ }^{9}$ to the simulations for $1 \leq-s_{\mathrm{ex}} / k_{\mathrm{B}} \leq 3$ and, also, show the extrapolation behavior of this curve outside of this region. Evidently, the relationship between $\lambda^{+}$and $-s_{\mathrm{ex}} /$ $k_{\mathrm{B}}$ is not even roughly exponential for the entire fluid domain. A much more accurate empirical model for the scaled thermal conductivity is presented in Section 9.3.

\section{ZERO-DENSITY LIMIT}

Carrying Rosenfeld's thoughts about the transport properties of dilute gases of finite density to their logical conclusion, we desire to understand the behavior of these scaled transport properties as the density goes to zero (the zero-density limit). In Rosenfeld's derivations, ${ }^{9}$ the dilute-gas transport properties were proportional to the excess entropy to the power of $-2 / 3$, but this formulation is invalid at zero excess entropy. Nonetheless, the scaling proposed in Section 7.1 has a welldefined zero-density limit, and it also results in another fascinating connection between transport properties and thermodynamic properties.

The zero-density transport properties of the Lennard-Jones 12-6 potential (to first order) are given by ${ }^{115,116}$

$$
\begin{aligned}
& {\left[\eta_{\rho \rightarrow 0}^{*}\right]_{1}=\frac{5}{16 \sqrt{\pi}} \frac{\sqrt{T^{*}}}{\Omega^{(2,2)^{*}}\left(T^{*}\right)}} \\
& {\left[\lambda_{\rho \rightarrow 0}^{*}\right]_{1}=\frac{15}{4}\left[\eta_{\rho \rightarrow 0}^{*}\right]_{1}} \\
& {\left[\left(\rho^{*} D^{*}\right)_{\rho \rightarrow 0}\right]_{1}=\frac{3 \sqrt{T^{*}}}{8 \sqrt{\pi} \Omega^{(1,1)^{*}}\left(T^{*}\right)}}
\end{aligned}
$$

and correction terms $\left(f_{\eta}^{(3)}, f_{\lambda}^{(3)}, f_{\mathrm{D}}^{(2)}\right)^{117}$ (all within $1.2 \%$ of unity in $\left.0.3 \leq T^{*} \leq 400\right)$ are available to bring the Sonine expansion up to higher order, as shown in the SI (Section 1.4). This correction yields

$$
\begin{aligned}
& \eta_{\rho \rightarrow 0}^{*}=\left[\eta_{\rho \rightarrow 0}^{*}\right]_{1} f_{\eta}^{(3)}\left(T^{*}\right) \\
& \lambda_{\rho \rightarrow 0}^{*}=\left[\lambda_{\rho \rightarrow 0}^{*}\right]_{1} f_{\lambda}^{(3)}\left(T^{*}\right) \\
& \left(\rho^{*} D^{*}\right)_{\rho \rightarrow 0}=\left[\left(\rho^{*} D^{*}\right)_{\rho \rightarrow 0}\right]_{1} f_{D}^{(2)}\left(T^{*}\right)
\end{aligned}
$$

In the case of $\eta^{+}$(approach is the same for all three transport coefficients), evaluation of the zero-density limit of $\eta^{+}$from eq 31 is undefined due to the fraction $(0 / 0)^{2 / 3}$. The rule of de l'Hôpital is used on the limit with a variable transformation (see the SI, Section 1.2) with a useful (and exact) intermediate result (this is the same result obtained from the derivative of eq 27) that

$$
\lim _{\rho^{*} \rightarrow 0}\left(\frac{\partial\left(-s_{\mathrm{ex}} / k_{\mathrm{B}}\right)}{\partial \rho^{*}}\right)_{T^{*}}=T^{*}\left(\frac{\mathrm{d} B_{2}^{*}}{\mathrm{~d} T^{*}}\right)+B_{2}^{*}
$$

resulting in

$$
\lim _{\rho_{\mathrm{N}} \rightarrow 0} \eta^{+}=\frac{\eta_{\rho \rightarrow 0}^{*}}{\sqrt{T^{*}}}\left[T^{*}\left(\frac{\mathrm{d} B_{2}^{*}}{\mathrm{~d} T^{*}}\right)+B_{2}^{*}\right]^{2 / 3}
$$

The approaches for thermal conductivity and self-diffusion are analogous, resulting in

$$
\begin{aligned}
& \lim _{\rho_{\mathrm{N}} \rightarrow 0} \lambda^{+}=\frac{\lambda_{\rho_{\mathrm{N}} \rightarrow 0}^{*}}{\sqrt{T^{*}}}\left[T^{*}\left(\frac{\mathrm{d} B_{2}^{*}}{\mathrm{~d} T^{*}}\right)+B_{2}^{*}\right]^{2 / 3} \\
& \lim _{\rho_{\mathrm{N}} \rightarrow 0} D^{+}=\frac{\left(\rho^{*} D^{*}\right)_{\rho_{\mathrm{N}} \rightarrow 0}}{\sqrt{T^{*}}}\left[T^{*}\left(\frac{\mathrm{d} B_{2}^{*}}{\mathrm{~d} T^{*}}\right)+B_{2}^{*}\right]^{2 / 3}
\end{aligned}
$$

8.1. Moderate Temperatures. For "reasonable" temperatures, the values of $\eta^{+}, \lambda^{+}$, and $D^{+}$in the zero-density limit may be obtained by leveraging the virial coefficients $B_{2}^{*}$ obtained from the equation of state of Thol et al. ${ }^{104}$ along with the correlations for the collision integrals of Kim and Monroe. ${ }^{115}$ Figure 10 shows the values of the Lennard-Jones

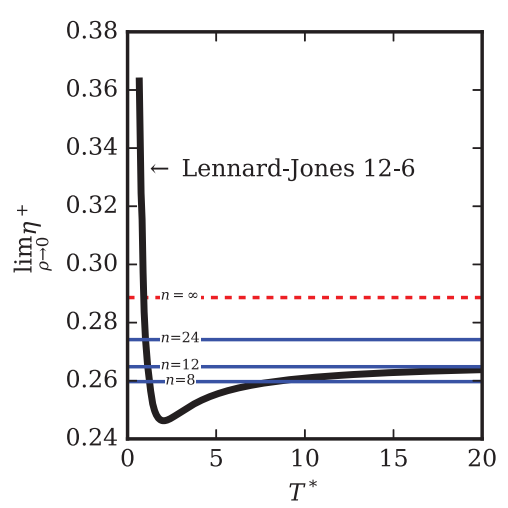

Figure 10. Zero-density limit of Lennard-Jones $12-6$ viscosity along with hard-sphere and inverse-power-law results. The IPL potentials of hardness $n$ are labeled in blue, and the hard-sphere value is shown in red.

12-6 potential along with the (constant) values for the hardsphere potential and selected inverse-power-law potentials. A similar figure for the thermal conductivity would be obtained by multiplying all values by roughly $15 / 4$ (deviation from 15/4 based on higher-order corrections differing between thermal conductivity and viscosity).

The second virial coefficient of the IPL potential is known exactly, and the virial coefficient term in the zero-density transport properties for the IPL potential can be obtained from (see the SI)

$$
T^{*}\left(\frac{\mathrm{d} B_{2}^{*}}{\mathrm{~d} T^{*}}\right)+B_{2}^{*}=\frac{2 \pi}{3} \Gamma(1-3 / n)(1-3 / n)\left(T^{*}\right)^{-3 / n}
$$

where $\Gamma$ is the Euler gamma function. Therefore, as shown in the SI, the value of the scaled viscosity in the zero-density limit for IPL potentials of hardness $n$ is equal to

$$
\lim _{\rho_{\mathrm{N}} \rightarrow 0} \eta_{\mathrm{IPL}}^{+}=F_{\eta, \mathrm{IPL}}(n)
$$

where $F_{\eta, \mathrm{IPL}}$ is a parameter that is only a function of $n$ (see the SI, eq 56). The SI provides tabulated values of $F_{\eta, \text { IPL }}$ (Table S5) as well as code in Python to carry out the necessary numerical integration to evaluate $F_{\eta, \mathrm{IPL}}$ (SI Section 4.4).

Note that this parameter has no temperature dependence because the temperature dependence of $\eta^{+}$for IPL potentials has been captured entirely by the second virial coefficient term. In the case of $n \rightarrow \infty$ (the hard-sphere limit), the value of $\eta^{+}$in the zero-density limit is given by 


$$
\lim _{\rho \rightarrow 0} \eta_{\mathrm{HS}}^{+}=\frac{5}{16 \sqrt{\pi}}\left(\frac{2 \pi}{3}\right)^{2 / 3}
$$

The approach for the self-diffusion is exactly analogous. Figure 11 shows the values of the Lennard-Jones $12-6$ potential

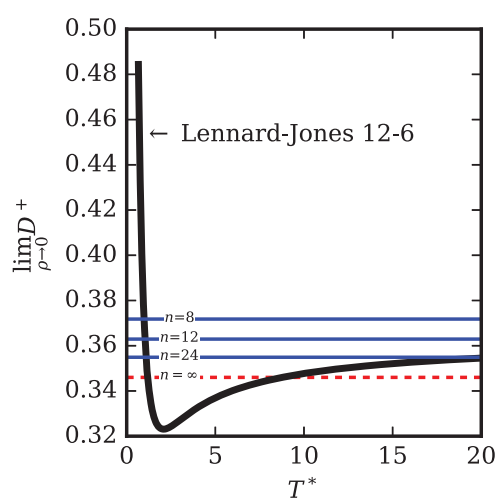

Figure 11. Zero-density limit of Lennard-Jones 12-6 self-diffusion along with hard-sphere and inverse-power-law results. The IPL potentials of hardness $n$ are labeled in blue, and the hard-sphere value is shown in red.

along with the (constant) values for the hard-sphere potential and selected inverse-power-law potentials. The value of $D^{+}$for the IPL potential in the zero-density limit is a unique function of the hardness of the IPL potential (has no temperature dependence) and is given in the SI (eq 59). The ratio of $D^{+} / \eta^{+}$ in the zero-density limit is in general defined as ${ }^{118}$

$$
\frac{D_{\rho \rightarrow 0}^{+}}{\eta_{\rho \rightarrow 0}^{+}}=\frac{6}{5} \frac{\Omega^{(2,2)^{*}}}{\Omega^{(1,1)^{*}}}
$$

In the case of the hard sphere, $\Omega_{\mathrm{HS}}^{(2,2) *} \equiv \Omega_{\mathrm{HS}}^{(1,1) *} \equiv 1$; thus, $D_{\mathrm{HS}}^{+} / \eta_{\mathrm{HS}}^{+}=6 / 5$, and

$$
\lim _{\rho \rightarrow 0} D_{\mathrm{HS}}^{+}=\frac{6}{16 \sqrt{\pi}}\left(\frac{2 \pi}{3}\right)^{2 / 3}
$$

The SI provides tabulated values of $F_{\mathrm{D}, \mathrm{IPL}}$ (Table S5) as well as code in Python to carry out the necessary numerical integration to evaluate $F_{\mathrm{D}, I P L}$ (SI Section 4.4).

8.2. High-Temperature Limit. At very high temperatures, the dilute Lennard-Jones 12-6 gas behaves like an inversepower-law potential with $n=12$ (see refs 14, 119, and 120) because at high temperatures only the large positive energies in the $r^{-12}$ repulsive term are significant compared to the thermal energies. As such, the high-temperature limits of the transport properties should approach those of the $n=12$ IPL.

Unfortunately, the high-temperature limit of the empirical models of Kim and Monroe ${ }^{115}$ and Thol et al., ${ }^{104}$ for the zerodensity-limit transport properties and second virial coefficient, respectively, are incorrect. This can be seen by consideration of each term individually. The respective figures are shown in the SI Appendix (Figures S11a,b and S12).

\section{SEMIEMPIRICAL MODELS}

Historically, highly accurate transport property models for a transport property $Y$ (where here $Y$ is one of $\eta^{+}, D^{+}$, or $\lambda^{+}$) have taken the form

$$
Y=Y_{\rho \rightarrow 0}+Y_{\mathrm{r}}+\Delta Y_{\text {crit }}
$$

in which $Y_{\rho \rightarrow 0}$ is the zero-density contribution, $Y_{\mathrm{r}}$ is the residual contribution, and $\Delta Y_{\text {crit }}$ is the critical enhancement contribution.

The motivation for the development of empirical correlations for the transport properties of the Lennard-Jones fluid is 3-fold:

1. We posit that the excess entropy is the parameter that determines the scaled transport properties (except for dilute gases). The deviations between correlation and simulation provide a means of quantifying the monovariability of this relationship.

2. Real molecules that are approximately spherical are frequently modeled as being like Lennard-Jones fluids, ${ }^{8}$ for which fitted values of $\varepsilon / k_{\mathrm{B}}$ and $\sigma$ are used to convert between physical units and Lennard-Jones units.

3. Many nonassociating real molecular fluids behave qualitatively like the Lennard-Jones fluid, and insight gained from developing correlations for the transport properties of the Lennard-Jones fluid can be directly applied to the transport property modeling of real fluids.

In order to assess the "goodness" of our models, we consider the conventional average absolute deviation $(\mathrm{AAD})$ of a property $Y$ defined by

$$
\mathrm{AAD}_{Y}=100 \times \frac{1}{N} \sum_{i=1}^{N}\left|\frac{Y_{\text {corr }, \mathrm{i}}}{Y_{\text {sim }, \mathrm{i}}}-1\right|
$$

In addition, while the term "uncertainty" is frequently used when discussing model fidelity, in this work we prefer to consider the distribution of deviations, and we define the term $U_{95}$ which is the central 95 percentiles of the signed deviation, from the 2.5 percentile to the 97.5 percentile. If the distribution of deviations were normally distributed, this would correspond roughly to two standard deviations above and below the mean.

9.1. Viscosity. In the case of viscosity, the critical enhancement term $\Delta Y_{\text {crit }}$ is usually ignored because there is only a very narrow region in the region of the critical point where the critical enhancement of viscosity is measurable. ${ }^{121}$ As such, engineering transport correlations usually involve a zero-density limit correlation and another for $Y_{r}$; we follow the same approach. The zero-density-limit is fully captured by the highly accurate collision integral analysis described above, and the zero-density reduced viscosity $\eta_{\rho \rightarrow 0}^{+}$is obtained by the combination of eq 44 and eq 40 along with the higher-order corrections described in the SI, Section 1.4.

Therefore, in order to develop the empirical correlation for viscosity, we first extract the residual viscosity by subtracting the zero-density-limit viscosity (all in reduced coordinates) for the primary data sets. In order to achieve a reasonable extrapolation behavior in the liquid region, the state points from Baidakov et al. ${ }^{69}$ at densities greater than values corresponding to the solidus line of van der Hoef ${ }^{78}$ were not included in the fit. As the residual reduced viscosity varies over a few decades, we would like to take the logarithm of the reduced viscosity, but the logarithm of a zero residual viscosity in the zero-density limit is mathematically problematic, so we add one to the residual viscosity. This results in a value that is mathematically well-defined from zero density to beyond the solidus line. The residual scaled viscosity is shown in Figure 12. Therefore, the empirical model for residual viscosity is expressed as the logarithm of $\eta^{+}-\eta_{\rho \rightarrow 0}^{+}+1$ as a function of 


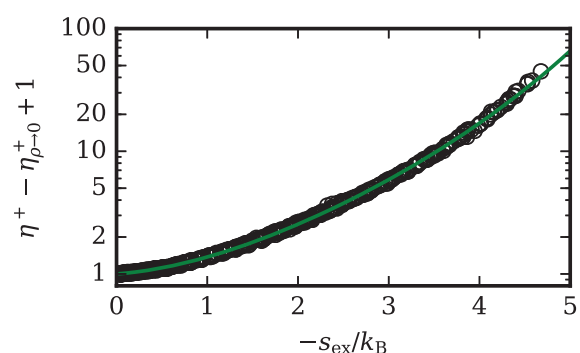

Figure 12. Scaled residual viscosity data from the primary data sets considered in the development of the correlation ${ }^{43-45}$ and the correlation from eq 54. Each marker corresponds to a single data point.

$-s_{\text {ex }} / k_{\mathrm{B}}$. A polynomial in reduced excess entropy is fit to the residual viscosity data with conventional least-squares polynomial fitting routines (the polyfit function in the numpy Python library).

This approach results in the functional form

$$
\eta_{\text {fit }}^{+}=\eta_{\rho \rightarrow 0}^{+}+\exp \left(\sum_{k=1}^{4} c_{\eta, k}\left(-s_{\mathrm{ex}} / k_{\mathrm{B}}\right)^{k}\right)-1
$$

Table 2. Coefficients for the Correlation in Equation 54

$\begin{array}{cc}k & c_{\eta, k} \\ 1 & 0.125364 \\ 2 & 0.220795 \\ 3 & -0.0313726 \\ 4 & 0.00313907\end{array}$

where the coefficients are in Table 2, and the zero-density viscosity is obtained from eq 41 . The value for $\eta^{*}$ is then obtained from

$$
\eta_{\mathrm{fit}}^{*}=\eta_{\mathrm{fit}}^{+} \frac{\left(\rho^{*}\right)^{2 / 3} \sqrt{T^{*}}}{\left(-s_{\mathrm{ex}} / k_{\mathrm{B}}\right)^{2 / 3}}
$$

where eq 43 can be used to evaluate $\left(-s_{\text {ex }} / k_{\mathrm{B}}\right) / \rho^{*}$ at zero density. Sample verification data are available in Table S6 in the SI.

Figure 13 shows a deviation plot of the correlation for viscosity along with the primary data sets, and Figure 14 shows the same figure for the secondary data sets. In general, the deviations of the model are within the uncertainty of the simulations. For instance the data of Meier et al. ${ }^{43}$ are estimated to have a statistical uncertainty of $10 \%$ in the gas phase, to $5 \%$ in the liquid phase, ${ }^{43}$ and a discussion of Meier's uncertainty analysis is provided elsewhere. ${ }^{65}$ The dilute-gas viscosity correlation is matched exactly, by construction. In the supercooled liquid, the data of Baidakov ${ }^{44}$ extend beyond the solidus line, and even for these very extreme states, the behavior of the correlation is reasonable; the liquid phase ends at an excess entropy of approximately $-s_{\mathrm{ex}} / k_{\mathrm{B}}=3.5$.

The secondary data sets are shown in Figure 14. These secondary data sets generally correspond to older simulations in which computational limitations of the day mandated a smaller number of particles or shorter simulation times. While the bulk of the data are still predicted within $10 \%$, there are some definite outliers. The data set of Heyes ${ }^{51}$ both is one of

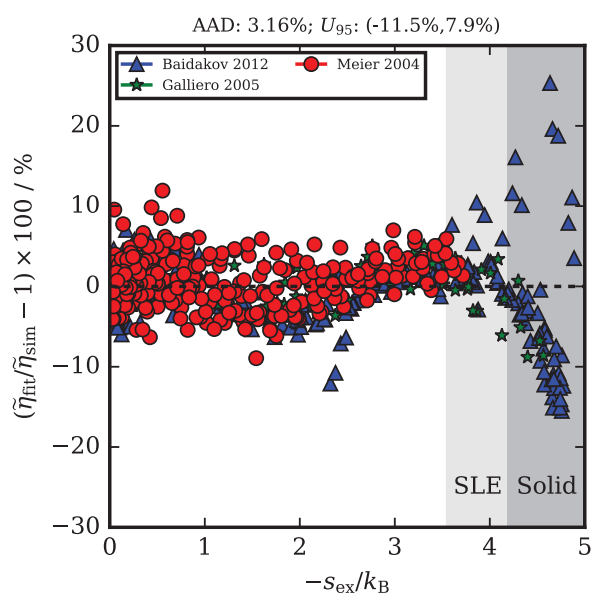

Figure 13. Deviation plot for the primary viscosity data sets ${ }^{43-45}$ compared with the correlation from eq 54. SLE refers to the approximate region of solid-liquid equilibrium, and solid to the approximate region of equilibrium solid.

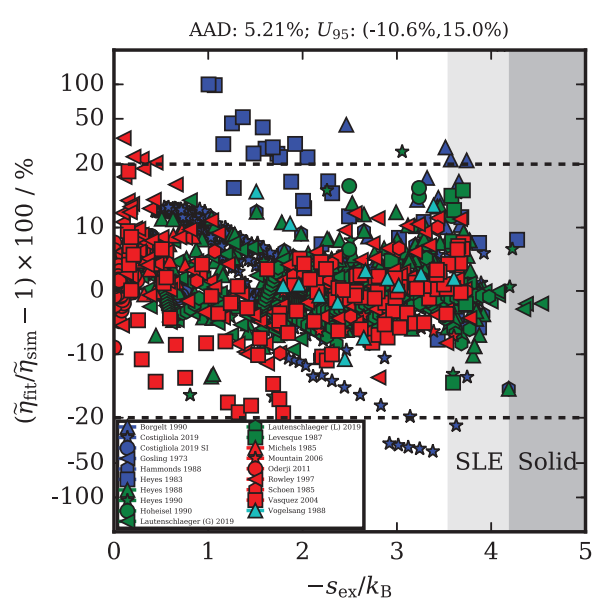

Figure 14. Deviation plot for the secondary viscosity data sets ${ }^{42,42,46-62}$ compared with the correlation from eq 54. The range of the ordinate from $-20 \%$ to $20 \%$ is linearly scaled, and logarithmically scaled outside this range. SLE refers to the approximate region of solid-liquid equilibrium, and solid to the approximate region of equilibrium solid.

the oldest simulations as well as systematically deviates from the bulk of the dense phase data.

An exceptional case is the study of Costigliola et al., ${ }^{47}$ in which simulations were carried out for some states with $T^{*}>$ 10000 (and many above $T^{*}>400$, in which case they were not included in further analysis according to our curation criteria above). Of the modern simulations, these data correspond to some of the largest deviations. The origin of these deviations is not currently well-understood. One hypothesis was that the equation of state of $\mathrm{Thol}^{104}$ provided erroneous values for the excess entropy at extreme temperatures. The EOS has a stated maximum temperature of $T^{*}=9$; however, confirmatory simulations of the excess entropy ${ }^{4,5}$ for temperatures exceeding $T^{*}=1000$ showed that the excess-entropy calculations were all within $5 \%$ of the predictions of the EOS of Thol. ${ }^{104} \mathrm{~A}$ second possible explanation is that excess-entropy scaling does not work at these extreme temperatures. As the temperature increases for a given density, the motion of the particles should become more and more dominated by repulsion, and the correlation (in the $R$-simple sense) should increase; this should 
in theory be a part of the phase diagram where excess-entropy scaling is the most successful. Further study is needed to investigate the origin of this breakdown in this scaling approach. This question is of rather more theoretical interest, as a temperature of $T^{*}=400$ corresponds to (for argon with an $\varepsilon / k_{\mathrm{B}}$ on the order of $100 \mathrm{~K}$ ) a temperature on the order of $40000 \mathrm{~K}$. Nonetheless, when the deviations between the data of ref 47 and the correlation are compared with those of other empirical models in the literature (see Section 9.4), it is clear that the excess-entropy scaling approach is by far the most successful in capturing the behavior of viscosity at extremely high temperatures.

9.2. Self-Diffusion. The self-diffusion represents a rather more challenging modeling problem than either viscosity or thermal conductivity. The impact of finite-size effects is rather significant, and the correction term in eq 22 can be as much as $20 \%$ of the value of the self-diffusion for some older data sets. In addition, the self-diffusion in the zero-density limit is larger in magnitude than the value in the dense phase, so subtracting off the dilute contribution is problematic.

In the entire fluid domain, the self-diffusion can be represented by

$$
D^{+}=(1-W) D_{\rho_{\mathrm{N}} \rightarrow 0}^{+}\left(T^{*}\right)+W D_{\text {dense }}^{+}\left(-s_{\mathrm{ex}} / k_{\mathrm{B}}\right)
$$

where $W$ is a parameter that crosses over from being approximately 0 in the zero-density to 1 in the dense phase. In this case we use a smoothed Heaviside step function given by

$$
W=\frac{1}{1+\exp \left[-\kappa_{\mathrm{D}}\left(\left(-s_{\mathrm{ex}} / k_{\mathrm{B}}\right)-\left(-s_{\mathrm{ex}} / k_{\mathrm{B}}\right)_{\text {cross }}\right)\right]}
$$

where $\kappa_{\mathrm{D}}$ is a parameter that controls the sharpness of the transition (the larger the $\kappa_{\mathrm{D}}$, the sharper the transition), and $\left(-s_{\mathrm{ex}} / k_{\mathrm{B}}\right)_{\text {cross }}$ is the center of the step function. Figure 15 shows

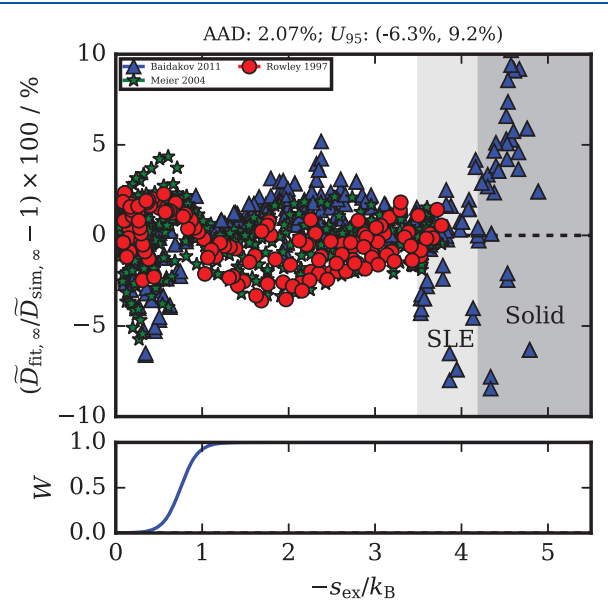

Figure 15. Deviation plot for the primary self-diffusion data sets $^{41,59,69}$ compared with the correlation from eq 58 and the smoothing function.

a graphical representation of this function. A similar kind of crossover approach has been previously used in empirical model development. ${ }^{122,123}$ The dense contribution is given by a polynomial in the excess entropy, in an analogous fashion to viscosity (Table 3 )

$$
D_{\text {dense }}^{+}=\sum_{k=0}^{4} c_{\mathrm{D}, k}\left(-s_{\mathrm{ex}} / k_{\mathrm{B}}\right)^{k}
$$

Table 3. Coefficients for the Correlation in Equation $58^{a}$

$\begin{array}{cc}k & c_{\mathrm{D}, k} \\ 0 & 0.342982 \\ 1 & 0.000201587 \\ 2 & -0.0428472 \\ 3 & 0.00783515 \\ 4 & -0.000362634\end{array}$

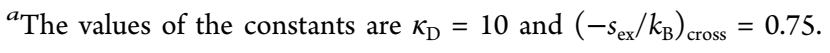

Figure 15 shows the deviations for the primary data sets. The claimed uncertainties from Meier et al. ${ }^{41}$ are on the order of $1 \%$ in the gas phase to $0.5 \%$ in the liquid phase, and the empirical model approaches, but does not quite reproduce, the data within these claimed uncertainties. Nonetheless, the extrapolation into the supercooled liquid is quite reasonable, and overall, the agreement with the primary data sets is acceptable. Figure 16 shows the same figure for the secondary

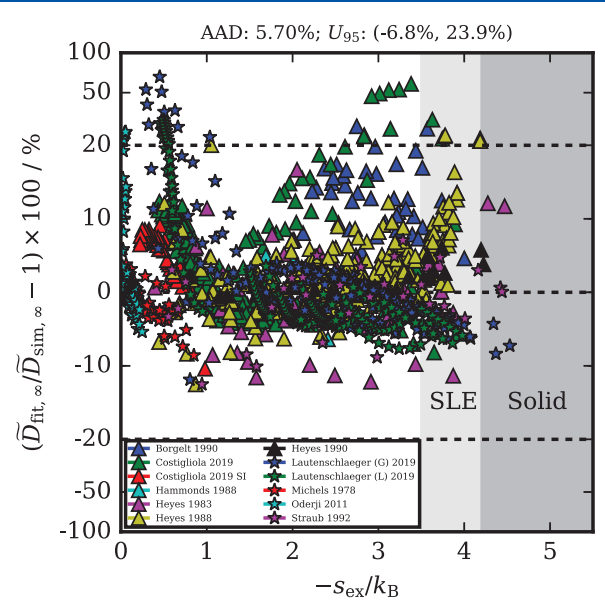

Figure 16. Deviation plot for the secondary self-diffusion data sets $42,42,46-48,50-53,58,70,71$ compared with the correlation from eq 58 . The range of the ordinate from $-20 \%$ to $20 \%$ is linearly scaled, and logarithmically scaled outside this range.

data sets. Here, too, simulation data are available up to temperatures of $T^{*}=400$ (and are also available at even higher temperatures that we did not include), and the very hightemperature data are still reproduced generally within $20 \%$. This stands in marked contrast to a number of the empirical models proposed in the literature (Section 9.4).

9.3. Thermal Conductivity. The modeling of thermal conductivity is more demanding than that of the viscosity because of the presence of critical enhancement. According to the state-of-the-art theory regarding the critical region, the thermal conductivity is infinite at the critical point and decays to its background value away from the critical point somewhat slowly. ${ }^{121}$ The viscosity, while also infinite at the critical point, decays to its background value much more rapidly, which is why the critical enhancement of viscosity was not included in Section 9.1. The critical enhancement complicates the application of excess-entropy scaling because isomorph theory is not expected to be valid in the vicinity of the critical point, ${ }^{124}$ and therefore, knowledge of the excess entropy 
should not be expected to be sufficient to predict the thermal conductivity. Indeed, as we will see, the critical enhancement of thermal conductivity is significant.

9.3.1. Critical Enhancement. For molecular fluids, the recent engineering reference correlations use the simplified critical enhancement of Olchowy and Sengers. ${ }^{121,125}$ In simulation units (see the SI, Section 1.5), the critical enhancement is given by

$$
\begin{aligned}
& \Delta_{\mathrm{c}} \lambda^{*}=\frac{R_{\mathrm{D}}}{6 \pi} \frac{\rho^{*} c_{p}^{*} T^{*}}{\eta^{*} \xi^{*}}\left(\Omega-\Omega_{0}\right) \\
& \Omega=\frac{2}{\pi}\left[\left(1-\kappa^{-1}\right) \arctan (y)+\kappa^{-1} y\right] \\
& \Omega_{0}=\frac{2}{\pi}\left[1-\exp \left(-\frac{1}{y^{-1}+(y / \delta)^{2} / 3}\right)\right] \\
& \Upsilon^{*}=\left(\frac{\partial \rho^{*}\left(T^{*}, \rho^{*}\right)}{\partial p^{*}}\right)_{T^{*}}-\frac{T_{\mathrm{R}}^{*}}{T^{*}}\left(\frac{\partial \rho^{*}\left(T_{\mathrm{R}}^{*}, \rho^{*}\right)}{\partial p^{*}}\right)_{T^{*}} \\
& \xi^{*}=\xi_{0}^{*}\left(\frac{p_{\mathrm{c}}^{*} \rho^{*}}{\Gamma_{\lambda}\left(\rho_{\mathrm{c}}^{*}\right)^{2}}\right)^{\nu_{\lambda} / \gamma_{\lambda}}\left[\Upsilon^{*}\right]^{\nu_{\lambda} / \gamma_{\lambda}}
\end{aligned}
$$

with $\kappa=c_{p}^{*} / c_{v}^{*}, y=\xi^{*} /\left(q_{\mathrm{D}}^{-1}\right)^{*}, \delta=\rho^{*} / \rho_{\mathrm{c}}^{*}$. The viscosity is obtained from eq 54. If $Y^{*}<0$, the critical enhancement term $\Delta_{\mathrm{c}} \lambda^{*}$ is set to zero. The quasiuniversal constants ${ }^{126}$ are given by $R_{\mathrm{D}}=1.02, \nu_{\lambda}=0.630$, and $\gamma_{\lambda}=1.239$. The values of $\Gamma_{\lambda}=$ $0.0496, \xi_{0}^{*}=1.2$, and $\left(q_{\mathrm{D}}^{-1}\right)^{*}=3.2$ were obtained by manual optimization.

Figure 17 shows the relative critical enhancement of thermal conductivity from each of the simulations of thermal

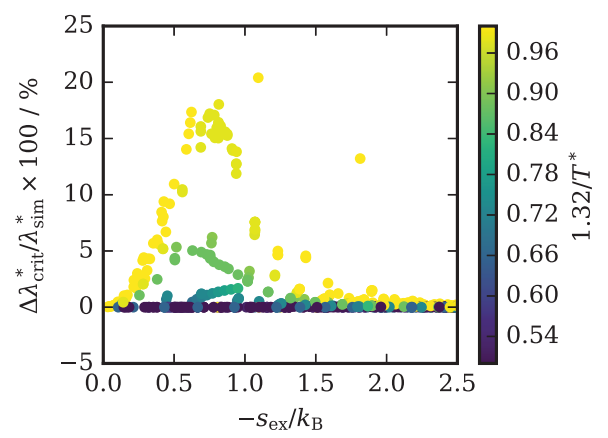

Figure 17. Critical enhancement of thermal conductivity as a function of excess entropy, where the critical enhancement is evaluated from eqs $59-63$.

conductivity. The critical enhancement is evaluated in eqs 59-63, with the thermodynamic parameters coming from the appropriate equation of state. ${ }^{104,114}$

9.3.2. Empirical Correlation. When both of the critical and zero-density contributions to the thermal conductivity are subtracted off, all that remains is the residual contribution, which is a nearly monovariate function of $-s_{\mathrm{ex}} / k_{\mathrm{B}}$, as can be seen in Figure 18.

The residual reduced thermal conductivity is defined by

$$
\lambda^{\mathrm{r},+} \equiv \lambda^{+}-\lambda_{\rho \rightarrow 0}^{+}-\Delta \lambda_{\text {crit }}^{+}
$$

and we fit the residual thermal conductivity by the polynomial

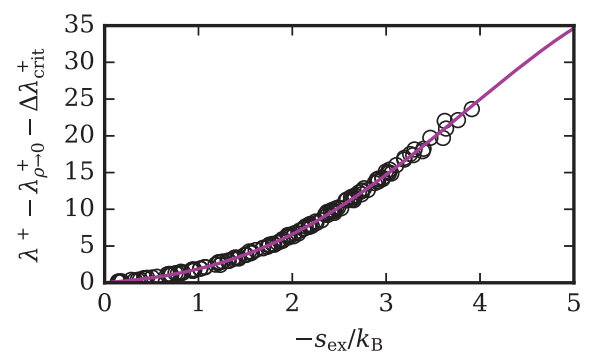

Figure 18. Scaled residual thermal conductivity data from the primary data sets ${ }^{57,63-65}$ considered in the development of the correlation and the correlation from eq 65. Each marker corresponds to a single data point.

$$
\lambda_{\mathrm{fit}}^{\mathrm{r},+}=\sum_{k=1}^{4} c_{\lambda, k}\left(-s_{\mathrm{ex}} / k_{\mathrm{B}}\right)^{k}
$$

Table 4. Coefficients for the Correlation in Equation 65

\begin{tabular}{cc}
$k$ & $c_{\lambda, k}$ \\
1 & 1.02796 \\
2 & 0.439252 \\
3 & 0.487628 \\
4 & -0.0679026 \\
\hline
\end{tabular}

where the coefficients are in Table 4. A similar fitting approach is used to that of the viscosity. The thermal conductivity is then obtained from

$$
\lambda^{*}=\frac{\left(\rho^{*}\right)^{2 / 3} \sqrt{T^{*}}}{\left(-s_{\mathrm{ex}} / k_{\mathrm{B}}\right)^{2 / 3}}\left(\lambda_{\mathrm{fit}}^{\mathrm{r},+}+\lambda_{\rho \rightarrow 0}^{+}+\Delta \lambda_{\text {crit }}^{+}\right)
$$

where, again, eq 43 can be used to evaluate $\left(-s_{\mathrm{ex}} / k_{\mathrm{B}}\right) / \rho^{*}$ at zero density.

Figure 19 shows a plot of the deviations between the simulation data and the correlation scheme for the primary data sets. In this case, the deviations between the correlation and the primary data sets are all less than $6 \%$. The data are

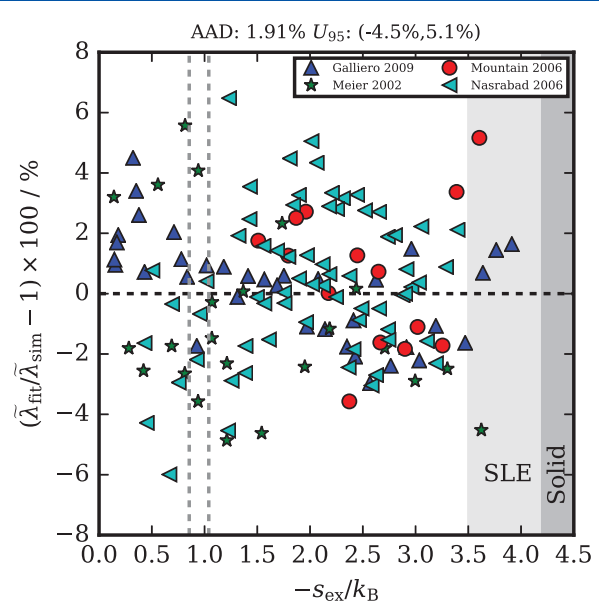

Figure 19. Deviation plot for the primary thermal conductivity data ${ }^{57,63-65}$ compared with the correlation from eq 65 . The dashed vertical lines indicate the excess entropy evaluated at the critical point of the LJTS and full Lennard-Jones 12-6 potentials. 
evenly distributed around the zero deviation line and are represented within their uncertainties. Unlike for viscosity, the data are mostly found in the homogeneous liquid phase, and only a few data points approach, or indeed enter, the supercooled domain.

Figure 20 shows the same figure for the secondary data sets. Aside from a few outliers, the data are all generally represented

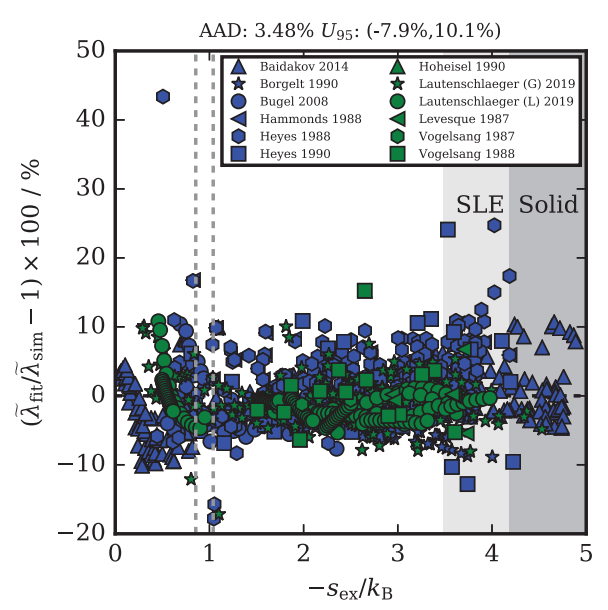

Figure 20. Deviation plot for the secondary thermal conductivity data sets ${ }^{42,46,50,52-55,62,66-68}$ compared with the correlation from eq 65 . The dashed vertical lines indicate the excess entropy evaluated at the critical point of the LJTS and full Lennard-Jones 12-6 potentials.

within $10 \%$. There are a few data points from Baidakov et al. ${ }^{66}$ that are in the supercooled $\left(-s_{\mathrm{ex}} / k_{\mathrm{B}} \gtrsim 4\right)$ region, and even those points are well-represented by the correlation. In the critical region, indicated by the two dashed lines, there are a few points that deviate more strongly from the correlation, but they are indeed outliers, and the general behavior of the correlation is a faithful representation of the data, even in the critical region and for supercooled states.

9.4. Model Comparison. There are several empirical models available in the literature for the transport properties of the Lennard-Jones fluid; Lautenschlaeger and Hasse ${ }^{42}$ provided a small review of the existing models. These empirical models were generally fit to the simulations carried out by the author who proposed the model. We summarize the average absolute relative deviation ( $\mathrm{AAD}$ ) of the models according to the primary and secondary data sets and list them in Table 5 . Additional figures are available in the SI (see SI Section 7) with deviation plots corresponding to each model.

For viscosity, the empirical model of Galliero and Boned is able to reproduce the simulation viscosity data with an AAD lower than the AAD of the correlation proposed in this work. Their empirical model has 6 adjustable parameters (ours has 4) but it suffers from poor extrapolation at extreme temperatures and, to a lesser extent, at high densities. The representation of the data of Costigliola et. $\mathrm{al}^{47}$ with the correlation of Galliero and Boned is much worse than our proposed model. The empirical model of Lautenschlaeger and Hasse $^{42}$ is able to reproduce their own data within $10 \%$, but it yields much larger errors in prediction for other data sets. As published, the correlation of Rowley and Painter has two typographical errors (as corrected in Woodcock ${ }^{128}$ and also fixed in our implementation in the SI); its extrapolation behavior into the supercooled liquid phase is erroneous, and results in quite poor predictions. The model of Woodcock ${ }^{128}$
Table 5. Average Absolute Deviation (AAD) of the Model Predictions According to the Primary and Secondary Data Sets $^{a}$

\begin{tabular}{llll} 
prop. & \multicolumn{1}{c}{1 st author } & \multicolumn{1}{c}{$\mathrm{AAD}_{1}$} & \multicolumn{1}{c}{$\mathrm{AAD}_{2}$} \\
$\eta^{*}$ & Galliero $^{45}$ & $3.0 \%$ & $8.48 \times 10^{7} \%$ \\
$\eta^{*}$ & Lautenschlaeger $^{42}$ & $19.8 \%$ & $18.5 \%$ \\
$\eta^{*}$ & Rowley $^{59}$ & $3.66 \times 10^{23} \%$ & $5.04 \times 10^{75} \%$ \\
$\lambda^{*}$ & Galliero $^{63}$ & $2.2 \%$ & $3.9 \%$ \\
$\lambda^{*}$ & Lautenschlaeger $^{42}$ & $3.2 \%$ & $3.9 \%$ \\
$\lambda^{*}$ & Bugel $^{67}$ & $4.1 \%$ & $6.8 \%$ \\
$\rho^{*} D^{*}$ & Lautenschlaeger $^{42}$ & $11.3 \%$ & $951.5 \%$ \\
$\rho^{*} D^{*}$ & Ruckenstein $^{127}$ & $12.4 \%$ & $12.3 \%$ \\
$\rho^{*} D^{*}$ & Rowley $^{59}$ & $667.7 \%$ & $153.0 \%$
\end{tabular}

${ }^{a} \mathrm{AAD}_{1}: \mathrm{AAD}$ for the primary data sets. $\mathrm{AAD}_{2}: \mathrm{AAD}$ for the secondary data sets.

could not be compared because working in concert with the author its typographical errors could not be corrected, but in general it produces similar predictions to those of ref 59. Lötgering-Lin et al. ${ }^{27}$ also provide a correlation for the Lennard-Jones viscosity, but it is only valid in a portion of the liquid phase.

Thermal conductivity is the transport property for which the models are in the best agreement. All three models in the literature yield AADs that are less than $5 \%$ for the primary data sets. The secondary data sets are predicted somewhat more poorly, just as is the case for our study. Again, the correlation of Galliero and Boned ${ }^{63}$ is the empirical model that comes closest to reproducing the $\mathrm{AAD}$ of the model presented in this work.

In the case of self-diffusion, the existing empirical models ${ }^{42,59,127}$ provide poor predictions of the finite-sizecorrected transport properties of the Lennard-Jones fluid. Two primary factors contribute to this unfortunate state of affairs:

- The correlations were fit to uncorrected self-diffusion data, and the finite-size correction shifts the self-diffusion data significantly for some of the older data sets.

- In the case of Rowley and Painter, ${ }^{59}$ the maximum temperature of the correlation is below the maximum temperature of the newer data set from Meier, ${ }^{41}$ and the extrapolation behavior of the correlation is poor.

9.5. Verification Data. A Python script is provided in the Supporting Information that implements the necessary parts of the equation of state of Thol et al. ${ }^{104}$ as well as the transport property correlations provided in this work. In order to ensure reproducibility of these results, sample values calculated from the correlations were programmatically generated from that script and are presented in Table S6 in the SI. In the event of discrepancies between the description in this work and the script, the script in the SI should be considered as the canonical source of information.

\section{CONCLUSIONS}

The empirical correlations developed in this work are based on the most comprehensive meta-study to date of the transport properties of the Lennard-Jones 12-6 model system, and they represent the most accurate transport property correlations published in the literature for the Lennard-Jones 12-6 fluid. These correlations demonstrate excellent extrapolation to extreme temperatures and densities. 
The condition of the Pearson coefficient $R$ between the virial energy and the potential energy being greater than 0.9 (the Roskilde simpleness condition) guarantees that entropy scaling will apply, but it does not appear to be a necessary condition. Considering the data in the gaseous phase, where the correlation parameter $R$ is much less than 0.9 (see Figure 4), the gas-phase data still collapse in the same way as the liquid phase.

The new scaling of viscosity is to a surprisingly good approximation given by $\eta^{+} \propto \exp \left(-s_{\mathrm{ex}} / k_{\mathrm{B}}\right)$ from the dilute-gas phase well into the supercooled liquid. Thermal conductivity and self-diffusion demonstrate a very strong correlation between the excess entropy and our novel scaled transport properties. We hope that these results will form the basis of new theoretical insights.

\section{ASSOCIATED CONTENT}

\section{S Supporting Information}

The Supporting Information is available free of charge on the ACS Publications website at DOI: 10.1021/acs.jpcb.9b05808.

Python implementation of the correlations for the transport properties proposed in this work (ZIP)

Python implementation of the literature correlations considered in this work (ZIP)

Complete set of simulation data collected from literature (ZIP)

Additional figures and analysis not suitable for inclusion into the main manuscript (PDF)

\section{AUTHOR INFORMATION}

\section{Corresponding Author}

*E-mail: ian.bell@nist.gov.

ORCID $\odot$

Ian H. Bell: 0000-0003-1091-9080

Monika Thol: 0000-0002-3250-0515

\section{Notes}

The authors declare no competing financial interest.

\section{ACKNOWLEDGMENTS}

The authors thank Ulrich Deiters (of the University of Cologne) for performing additional Monte Carlo simulations on the CHEOPS supercomputer of the University of Cologne, Martin Lautenschlaeger (of the University of Kaiserslautern) for helpful discussions and carrying out the literature survey that formed the basis of the literature survey used in this study, and Arno Laesecke (formerly of NIST) for highlighting the importance of the Lennard-Jones fluid and initial data collection. This work was supported by the VILLUM Foundation's Matter grant (16515).

\section{REFERENCES}

(1) Rosenfeld, Y. Relation between the Transport Coefficients and the Internal Entropy of Simple Systems. Phys. Rev. A: At., Mol., Opt. Phys. 1977, 15, 2545-2549.

(2) Bell, I. H. Probing the Link between Residual Entropy and Viscosity of Molecular Fluids and Model Potentials. Proc. Natl. Acad. Sci. U. S. A. 2019, 116, 4070-4079.

(3) Bell, I. H.; Laesecke, A. Viscosity of Refrigerants and Other Working Fluids from Residual Entropy Scaling. 16th International Refrigeration and Air Conditioning Conference at Purdue, July 11-14, 2016; 2016; p 2287.
(4) Detmar, E.; Yazdi Nezhad, S.; Deiters, U. K. Determination of the Residual Entropy of Simple Mixtures by Monte Carlo Simulation. Langmuir 2017, 33, 11603-11610.

(5) Nezhad, S. Y.; Deiters, U. K. Estimation of the Entropy of Fluids with Monte Carlo Computer Simulation. Mol. Phys. 2017, 115, 10741085.

(6) O'Connell, J. P.; Haile, J. Thermodynamics: Fundamentals for Applications; Cambridge University Press: New York, 2005.

(7) Marsh, K. N. Editorial. J. Chem. Eng. Data 1997, 42, 1.

(8) Rutkai, G.; Thol, M.; Span, R.; Vrabec, J. How Well Does the Lennard-Jones Potential Represent the Thermodynamic Properties of Noble Gases? Mol. Phys. 2017, 115, 1104-1121.

(9) Rosenfeld, Y. A. Quasi-universal Scaling Law for Atomic Transport in Simple Fluids. J. Phys.: Condens. Matter 1999, 11, 54155427.

(10) Andrade, E. N. Da C. The Viscosity of Liquids. Nature 1930, $125,582-584$.

(11) Andrade, E. N. Da C. Viscosity of Liquids. Nature 1931, 128, 835-835.

(12) Andrade, da C.; XLI, E. a Theory of the Viscosity of Liquids. Part I. London, Edinburgh, and Dublin Philosophical Magazine and Journal of Science 1934, 17, 497-511.

(13) Dyre, J. C. Perspective: Excess-Entropy Scaling. J. Chem. Phys. 2018, 149, 210901.

(14) Pedersen, U. R.; Bailey, N. P.; Schrøder, T. B.; Dyre, J. C. Strong Pressure-Energy Correlations in van der Waals Liquids. Phys. Rev. Lett. 2008, 100, 015701.

(15) Gnan, N.; Schrøder, T. B.; Pedersen, U. R.; Bailey, N. P.; Dyre, J. C. Pressure-Energy Correlations in Liquids. IV. "Isomorphs" in Liquid Phase Diagrams. J. Chem. Phys. 2009, 131, 234504.

(16) Dyre, J. C. Hidden Scale Invariance in Condensed Matter. J. Phys. Chem. B 2014, 118, 10007-10024.

(17) Schrøder, T. B.; Dyre, J. C. Simplicity of Condensed Matter at Its Core: Generic Definition of a Roskilde-Simple System. J. Chem. Phys. 2014, 141, 204502.

(18) Dyre, J. C. Simple Liquids' Quasiuniversality and the HardSphere Paradigm. J. Phys.: Condens. Matter 2016, 28, 323001.

(19) Ingebrigtsen, T. S.; Schrøder, T. B.; Dyre, J. C. What Is a Simple Liquid? Phys. Rev. X 2012, 2, 011011.

(20) Chapman, S.; Cowling, T. G. The Mathematical Theory of NonUniform Gases: An Account of the Kinetic Theory of Viscosity, Thermal Conduction and Diffusion in Gases; Cambridge University Press, 1970.

(21) Galliero, G.; Boned, C.; Fernández, J. Scaling of the Viscosity of the Lennard-Jones Chain Fluid Model, Argon, and Some Normal Alkanes. J. Chem. Phys. 2011, 134, 064505.

(22) Novak, L. T. Fluid Viscosity-Residual Entropy Correlation. Int. J. Chem. React. Eng. 2011, 9, A107.

(23) Novak, L. T. Self-Diffusion Coefficient and Viscosity in Fluids. Int. J. Chem. React. Eng. 2011, 9, A63.

(24) Novak, L. T. Predicting Natural Gas Viscosity with a Mixture Viscosity Model for the Entire Fluid Region. Ind. Eng. Chem. Res. 2013, 52, 16014-16018

(25) Novak, L. T. Predicting Fluid Viscosity of Nonassociating Molecules. Ind. Eng. Chem. Res. 2015, 54, 5830-5835.

(26) Lötgering-Lin, O.; Gross, J. Group Contribution Method for Viscosities Based on Entropy Scaling Using the Perturbed-chain Polar Statistical Associating Fluid Theory. Ind. Eng. Chem. Res. 2015, 54, $7942-7952$.

(27) Lötgering-Lin, O.; Fischer, M.; Hopp, M.; Gross, J. Pure Substance and Mixture Viscosities Based on Entropy Scaling and an Analytic Equation of State. Ind. Eng. Chem. Res. 2018, 57, 4095-4114.

(28) Hopp, M.; Gross, J. Thermal Conductivity of Real Substances from Excess Entropy Scaling Using PCP-SAFT. Ind. Eng. Chem. Res. 2017, 56, 4527-4538.

(29) Krekelberg, W. P.; Pond, M. J.; Goel, G.; Shen, V. K.; Errington, J. R.; Truskett, T. M. Generalized Rosenfeld Scalings for Tracer Diffusivities in Not-So-Simple Fluids: Mixtures and Soft Particles. Phys. Rev. E 2009, 80, 061205. 
(30) Krekelberg, W. P.; Kumar, T.; Mittal, J.; Errington, J. R.; Truskett, T. M. Anomalous Structure and Dynamics of the GaussianCore Fluid. Phys. Rev. E 2009, 79, 031203.

(31) Pond, M. J.; Errington, J. R.; Truskett, T. M. Communication: Generalizing Rosenfeld's Excess-Entropy Scaling to Predict LongTime Diffusivity in Dense Fluids of Brownian Particles: From Hard to Ultrasoft Interactions. J. Chem. Phys. 2011, 134, 081101.

(32) Bacher, A. K.; Schrøder, T. B.; Dyre, J. C. The EXP PairPotential System. II. Fluid Phase Isomorphs. J. Chem. Phys. 2018, 149, 114502.

(33) Bacher, A. K.; Schrøder, T. B.; Dyre, J. C. The EXP PairPotential System. I. Fluid Phase Isotherms, Isochores, and Quasiuniversality. J. Chem. Phys. 2018, 149, 114501.

(34) Eisenschitz, R.; London, F. Über Das Verhältnis Der Van Der Waalsschen Kräfte $\mathrm{Zu}$ Den Homöopolaren Bindungskräften. Eur. Phys. J. A 1930, 60, 491-527.

(35) London, F. The General Theory of Molecular Forces. Trans. Faraday Soc. 1937, 33, 8b-26.

(36) Hoang, H.; Delage-Santacreu, S.; Galliero, G. Simultaneous Description of Equilibrium, Interfacial, and Transport Properties of Fluids Using a Mie Chain Coarse-Grained Force Field. Ind. Eng. Chem. Res. 2017, 56, 9213-9226.

(37) Jaeger, F.; Matar, O. K.; Muller, E. A. Bulk Viscosity of Molecular Fluids. J. Chem. Phys. 2018, 148, 174504.

(38) Galliéro, G.; Boned, C.; Baylaucq, A.; Montel, F. Molecular Dynamics Comparative Study of Lennard-Jones $\alpha-6$ and Exponential $\alpha-6$ Potentials: Application to Real Simple Fluids (Viscosity and Pressure). Phys. Rev. E 2006, 73, 061201.

(39) Lafitte, T.; Apostolakou, A.; Avendaño, C.; Galindo, A.; Adjiman, C. S.; Müller, E. A.; Jackson, G. Accurate Statistical Associating Fluid Theory for Chain Molecules Formed from Mie Segments. J. Chem. Phys. 2013, 139, 154504.

(40) Mohr, P. J.; Newell, D. B.; Taylor, B. N.; Tiesinga, E. Data and Analysis for the CODATA 2017 Special Fundamental Constants Adjustment. Metrologia 2018, 55, 125-146.

(41) Meier, K.; Laesecke, A.; Kabelac, S. Transport Coefficients of the Lennard-Jones Model Fluid. II Self-Diffusion. J. Chem. Phys. 2004, 121, 9526-9535.

(42) Lautenschlaeger, M. P.; Hasse, H. Transport Properties of the Lennard-Jones Truncated and Shifted Fluid from Non-Equilibrium Molecular Dynamics Simulations. Fluid Phase Equilib. 2019, 482, 3847.

(43) Meier, K.; Laesecke, A.; Kabelac, S. Transport Coefficients of the Lennard-Jones Model Fluid. I. Viscosity. J. Chem. Phys. 2004, 121, 3671 .

(44) Baidakov, V. G.; Protsenko, S. P.; Kozlova, Z. R. Metastable Lennard-Jones Fluids. I. Shear Viscosity. J. Chem. Phys. 2012, 137, 164507.

(45) Galliéro, G.; Boned, C.; Baylaucq, A. Molecular Dynamics Study of the Lennard-Jones Fluid Viscosity: Application to Real Fluids. Ind. Eng. Chem. Res. 2005, 44, 6963-6972.

(46) Borgelt, P.; Hoheisel, C.; Stell, G. Exact Molecular Dynamics and Kinetic Theory Results for Thermal Transport Coefficients of the Lennard-Jones Argon Fluid in a Wide Region of States. Phys. Rev. A: At., Mol., Opt. Phys. 1990, 42, 789-794.

(47) Costigliola, L.; Heyes, D. M.; Schrøder, T. B.; Dyre, J. C. Revisiting the Stokes-Einstein Relation without a Hydrodynamic Diameter. J. Chem. Phys. 2019, 150, 021101.

(48) Costigliola, L. RUC Glass and Time Data Repository; 2019; http://glass.ruc.dk/data/ (Accessed January 14, 2019).

(49) Gosling, E. M.; McDonald, I.; Singer, K. On the Calculation by Molecular Dynamics of the Shear Viscosity of a Simple Fluid. Mol. Phys. 1973, 26, 1475-1484.

(50) Hammonds, K. D.; Heyes, D. M. Transport Coefficients of Model Simple Liquids. A Molecular-Dynamics Study and Effective Hard-Sphere Analysis. J. Chem. Soc., Faraday Trans. 2 1988, 84, 705725 .
(51) Heyes, D. M. Self-Diffusion and Shear Viscosity of Simple Fluids. A Molecular-Dynamics Study. J. Chem. Soc., Faraday Trans. 2 1983, 79, 1741-1758.

(52) Heyes, D. M. Transport Coefficients of Lennard-Jones Fluids: A Molecular-Dynamics and Effective-Hard-Sphere Treatment. Phys. Rev. B: Condens. Matter Mater. Phys. 1988, 37, 5677-5696.

(53) Heyes, D.; Powles, J. Information Theory Applied to the Transport Coefficients of Lennard-Jones Fluids. Mol. Phys. 1990, 71, $781-800$

(54) Hoheisel, C. Memory Functions and the Calculation of Dynamical Properties of Atomic Liquids. Comput. Phys. Rep. 1990, 12, 29-66.

(55) Levesque, D.; Verlet, L. Molecular Dynamics Calculations of Transport Coefficients. Mol. Phys. 1987, 61, 143-159.

(56) Michels, J.; Trappeniers, N. Molecular Dynamical Calculations of the Viscosity of Lennard-Jones Systems. Phys. A 1985, 133, 281290

(57) Mountain, R. D. System Size and Control Parameter Effects in Reverse Perturbation Nonequilibrium Molecular Dynamics. J. Chem. Phys. 2006, 124, 104109.

(58) Oderij, H. Y.; Ding, H.; Behnejad, H. Calculation of the Second Self-Diffusion and Viscosity Virial Coefficients of Lennard-Jones Fluid by Equilibrium Molecular Dynamics Simulations. Phys. Rev. E 2011, 83, 061202 .

(59) Rowley, R. L.; Painter, M. M. Diffusion and Viscosity Equations of State for a Lennard-Jones Fluid Obtained from Molecular Dynamics Simulations. Int. J. Thermophys. 1997, 18, 1109-1121.

(60) Schoen, M.; Hoheisel, C. The Shear Viscosity of a LennardJones Fluid Calculated by Equilibrium Molecular Dynamics. Mol. Phys. 1985, 56, 653-672.

(61) Vasquez, V. R.; Macedo, E. A.; Zabaloy, M. S. Lennard-Jones Viscosities in Wide Ranges of Temperature and Density: Fast Calculations Using a Steady-State Periodic Perturbation Method. Int. J. Thermophys. 2004, 25, 1799-1818.

(62) Vogelsang, R.; Hoheisel, G.; Luckas, M. Shear Viscosity and Thermal Conductivity of the Lennard-Jones Liquid Computed Using Molecular Dynamics and Predicted by a Memory Function Model for a Large Number of States. Mol. Phys. 1988, 64, 1203-1213.

(63) Galliero, G.; Boned, C. Thermal Conductivity of the LennardJones Chain Fluid Model. Phys. Rev. E 2009, 80, 061202.

(64) Nasrabad, A. E.; Laghaei, R.; Eu, B. C. Molecular Theory of Thermal Conductivity of the Lennard-Jones Fluid. J. Chem. Phys. 2006, 124, 084506.

(65) Meier, K. Computer Simulation and Interpretation of the Transport Coefficients of the Lennard-Jones Model Fluid. Ph.D. Thesis, University of the Federal Armed Forces Hamburg, 2002.

(66) Baidakov, V. G.; Protsenko, S. P. Metastable Lennard-Jones Fluids. II. Thermal Conductivity. J. Chem. Phys. 2014, 140, 214506.

(67) Bugel, M.; Galliero, G. Thermal Conductivity of the LennardJones Fluid: An Empirical Correlation. Chem. Phys. 2008, 352, 249257.

(68) Vogelsang, R.; Hoheisel, C.; Ciccotti, G. Thermal Conductivity of the Lennard-Jones Liquid by Molecular Dynamics Calculations. J. Chem. Phys. 1987, 86, 6371-6375.

(69) Baidakov, V.; Protsenko, S.; Kozlova, Z. The Self-Diffusion Coefficient in Stable and Metastable States of the Lennard-Jones Fluid. Fluid Phase Equilib. 2011, 305, 106-113.

(70) Michels, J.; Trappeniers, N. The Self-Diffusion Coefficient in the Gas Phase at Moderate Densities, Obtained by Computer Simulations. Phys. A 1978, 90, 179-195.

(71) Straub, J. E. Analysis of the Role of Attractive Forces in SelfDiffusion of a Simple Fluid. Mol. Phys. 1992, 76, 373-385.

(72) Albrechtsen, D. E.; Olsen, A. E.; Pedersen, U. R.; Schrøder, T. B.; Dyre, J. C. Isomorph Invariance of the Structure and Dynamics of Classical Crystals. Phys. Rev. B: Condens. Matter Mater. Phys. 2014, 90, 094106.

(73) Ingebrigtsen, T. S.; Schrøder, T. B.; Dyre, J. C. Isomorphs in Model Molecular Liquids. J. Phys. Chem. B 2012, 116, 1018-1034. 
(74) Dyre, J. C. Isomorphs, Hidden Scale Invariance, and Quasiuniversality. Phys. Rev. E 2013, 88, 042139.

(75) Mausbach, P.; Köster, A.; Vrabec, J. Liquid State Isomorphism, Rosenfeld-Tarazona Temperature Scaling, and Riemannian Thermodynamic Geometry. Phys. Rev. E: Stat. Phys., Plasmas, Fluids, Relat. Interdiscip. Top. 2018, 97, 052149.

(76) Schrøder, T. B.; Gnan, N.; Pedersen, U. R.; Bailey, N. P.; Dyre, J. C. Pressure-Energy Correlations in Liquids. V. Isomorphs in Generalized Lennard-Jones Systems. J. Chem. Phys. 2011, 134, 164505.

(77) Bailey, N. P.; Pedersen, U. R.; Gnan, N.; Schrøder, T. B.; Dyre, J. C. Pressure-Energy Correlations in Liquids. II. Analysis and Consequences. J. Chem. Phys. 2008, 129, 184508.

(78) van der Hoef, M. A. Free Energy of the Lennard-Jones Solid. J. Chem. Phys. 2000, 113, 8142-8148.

(79) Ahmed, A.; Sadus, R. J. Solid-Liquid Equilibria and Triple Points of $n$-6 Lennard-Jones Fluids. J. Chem. Phys. 2009, 131, 174504.

(80) Ahmed, A.; Sadus, R. J. Erratum: "Solid-Liquid Equilibria and Triple Points of $n-6$ Lennard-Jones Fluids" [J. Chem. Phys. 131, 174504 (2009)]. J. Chem. Phys. 2010, 133, 229902.

(81) Allen, M. P.; Tildesley, D. J. Computer Simulation of Liquids, 2nd ed.; Oxford University Press: Oxford England, 2017; pp xix, 385.

(82) Evans, D. J.; Morriss, G. P. Statistical Mechanics of Nonequilibrium Liquids; Academic Press, 1990; p 302.

(83) Maginn, E. J.; Messerly, R. A.; Carlson, D. J.; Roe, D. R.; Elliott, J. R. Best Practices for Computing Transport Properties 1. Selfdiffusivity and Viscosity from Equilibrium Molecular Dynamics [article V1.0]. Liv. J. Comp. Mol. Sci. 2019, 1, 6324.

(84) Lautenschlaeger, M. P.; Horsch, M.; Hasse, H. Simultaneous Determination of Thermal Conductivity and Shear Viscosity Using Two-Gradient Non-Equilibrium Molecular Dynamics Simulations. Mol. Phys. 2019, 117, 189-199.

(85) Hess, B. Determining the Shear Viscosity of Model Liquids from Molecular Dynamics Simulations. J. Chem. Phys. 2002, 116, 209-217.

(86) Hafskjold, B.; Ikeshoji, T.; Ratkje, S. K. On the Molecular Mechanism of Thermal Diffusion in Liquids. Mol. Phys. 1993, 80, $1389-1412$.

(87) Evans, D. J. Homogeneous NEMD Algorithm for Thermal Conductivity-Application of Non-Canonical Linear Response Theory. Phys. Lett. A 1982, 91, 457-460.

(88) Evans, M.; Heyes, D. Combined Shear and Elongational Flow by Non-Equilibrium Molecular Dynamics. Mol. Phys. 1990, 69, 241263.

(89) Müller-Plathe, F. A Simple Nonequilibrium Molecular Dynamics Method for Calculating the Thermal Conductivity. J. Chem. Phys. 1997, 106, 6082-6085.

(90) Müller-Plathe, F. Reversing the Perturbation in Nonequilibrium Molecular Dynamics: An Easy Way to Calculate the Shear Viscosity of Fluids. Phys. Rev. E: Stat. Phys., Plasmas, Fluids, Relat. Interdiscip. Top. 1999, 59, 4894-4898.

(91) Bedrov, D.; Smith, G. D. Thermal Conductivity of Molecular Fluids from Molecular Dynamics Simulations: Application of a New Imposed-Flux Method. J. Chem. Phys. 2000, 113, 8080-8084.

(92) Chen, T.; Smit, B.; Bell, A. T. Are Pressure Fluctuation-based Equilibrium Methods Really Worse Than Nonequilibrium Methods for Calculating Viscosities? J. Chem. Phys. 2009, 131, 246101.

(93) Berendsen, H. J. C.; Postma, J. P. M.; van Gunsteren, W. F.; DiNola, A.; Haak, J. R. Molecular Dynamics with Coupling to an External Bath. J. Chem. Phys. 1984, 81, 3684-3690.

(94) Bussi, G.; Donadio, D.; Parrinello, M. Canonical Sampling through Velocity Rescaling. J. Chem. Phys. 2007, 126, 014101.

(95) Nosé, S. A Unified Formulation of the Constant Temperature Molecular Dynamics Methods. J. Chem. Phys. 1984, 81, 511-519.

(96) Andersen, H. C. Molecular Dynamics Simulations at Constant Pressure and/or Temperature. J. Chem. Phys. 1980, 72, 2384-2393.

(97) Hoover, W. G. Canonical Dynamics: Equilibrium Phase-Space Distributions. Phys. Rev. A: At., Mol., Opt. Phys. 1985, 31, 1695-1697.
(98) Basconi, J. E.; Shirts, M. R. Effects of Temperature Control Algorithms on Transport Properties and Kinetics in Molecular Dynamics Simulations. J. Chem. Theory Comput. 2013, 9, 2887-2899.

(99) Yeh, I.-C.; Hummer, G. System-Size Dependence of Diffusion Coefficients and Viscosities from Molecular Dynamics Simulations with Periodic Boundary Conditions. J. Phys. Chem. B 2004, 108, $15873-15879$.

(100) Jamali, S. H.; Hartkamp, R.; Bardas, C.; Söhl, J.; Vlugt, T. J. H.; Moultos, O. A. Shear Viscosity Computed from the Finite-Size Effects of Self-diffusivity in Equilibrium Molecular Dynamics. J. Chem. Theory Comput. 2018, 14, 5959-5968.

(101) Merz, P. T.; Shirts, M. R. Testing for Physical Validity in Molecular Simulations. PLoS One 2018, 13, e0202764.

(102) Span, R. Multiparameter Equations of State-An Accurate Source of Thermodynamic Property Data; Springer, 2000.

(103) Ref 102, eq 3.31.

(104) Thol, M.; Rutkai, G.; Köster, A.; Lustig, R.; Span, R.; Vrabec, J. Equation of State for the Lennard-Jones Fluid. J. Phys. Chem. Ref. Data 2016, 45, 023101.

(105) Rutkai, G.; Thol, M.; Lustig, R.; Span, R.; Vrabec, J. Communication: Fundamental Equation of State Correlation with Hybrid Data Sets. J. Chem. Phys. 2013, 139, 041102.

(106) Lustig, R. Statistical Analogues for Fundamental Equation of State Derivatives. Mol. Phys. 2012, 110, 3041-3052.

(107) Lustig, R. Direct Molecular NVT Simulation of the Isobaric Heat Capacity, Speed of Sound and Joule-Thomson Coefficient. Mol. Simul. 2011, 37, 457-465.

(108) Lemmon, E. W.; Jacobsen, R. T. A New Functional Form and New Fitting Techniques for Equations of State with Application to Pentafluoroethane (HFC-125). J. Phys. Chem. Ref. Data 2005, 34, 69-108.

(109) Johnson, J. K.; Zollweg, J. A.; Gubbins, K. E. The LennardJones Equation of State Revisited. Mol. Phys. 1993, 78, 591-618.

(110) Kolafa, J.; Nezbeda, I. The Lennard-Jones Fluid: An Accurate Analytic and Theoretically-Based Equation of State. Fluid Phase Equilib. 1994, 100, 1-34.

(111) May, H.-O.; Mausbach, P. Riemannian Geometry Study of Vapor-Liquid Phase Equilibria and Supercritical Behavior of the Lennard-Jones Fluid. Phys. Rev. E 2012, 85, 031201.

(112) Mecke, M.; Müller, A.; Winkelmann, J.; Vrabec, J.; Fischer, J.; Span, R.; Wagner, W. An Accurate van der Waals-type Equation of State for the Lennard-Jones Fluid. Int. J. Thermophys. 1996, 17, 391404.

(113) Pieprzyk, S.; Brańka, A. C.; Maćkowiak, S.; Heyes, D. M. Comprehensive Representation of the Lennard-Jones Equation of State Based on Molecular Dynamics Simulation Data. J. Chem. Phys. 2018, 148, 114505

(114) Thol, M.; Rutkai, G.; Span, R.; Vrabec, J.; Lustig, R. Equation of State for the Lennard-Jones Truncated and Shifted Model Fluid. Int. J. Thermophys. 2015, 36, 25-43.

(115) Kim, S. U.; Monroe, C. W. High-accuracy Calculations of Sixteen Collision Integrals for Lennard-Jones (12-6) Gases and Their Interpolation to Parameterize Neon, Argon, and Krypton. J. Comput. Phys. 2014, 273, 358-373.

(116) Hirschfelder, J. O.; Curtiss, C. F.; Bird, R. B. Molecular Theory of Gases and Liquids; John Wiley and Sons, 1954.

(117) Ref 116, Table 1-P.

(118) Ref 116, eqs 8.2-48.

(119) Hansen, J.-P. Phase Transition of the Lennard-Jones System. II. High-Temperature Limit. Phys. Rev. A: At., Mol., Opt. Phys. 1970, 2, 221-230.

(120) Heyes, D. M.; Rickayzen, G.; Pieprzyk, S.; Brańka, A. C. The Second Virial Coefficient and Critical Point Behavior of the Mie Potential. J. Chem. Phys. 2016, 145, 084505.

(121) Perkins, R. A.; Sengers, J. V.; Abdulagatov, I. M.; Huber, M. L. Simplified Model for the Critical Thermal-Conductivity Enhancement in Molecular Fluids. Int. J. Thermophys. 2013, 34, 191-212.

(122) Bell, I. H.; Groll, E. A.; Braun, J. E.; Horton, W. T. A Computationally Efficient Hybrid Leakage Model for Positive 
Displacement Compressors and Expanders. Int. J. Refrig. 2013, 36, 1965-1973.

(123) Xiang, H. W.; Laesecke, A.; Huber, M. L. A New Reference Correlation for the Viscosity of Methanol. J. Phys. Chem. Ref. Data 2006, 35, 1597-1620.

(124) Costigliola, L.; Schrøder, T. B.; Dyre, J. C. Communication: Studies of the Lennard-Jones Fluid in 2, 3, and 4 Dimensions Highlight the Need for a Liquid-State 1/d Expansion. J. Chem. Phys. 2016, 144, 231101.

(125) Olchowy, G. A.; Sengers, J. V. A Simplified Representation for the Thermal Conductivity of Fluids in the Critical Region. Int. J. Thermophys. 1989, 10, 417-426.

(126) Ref 121, eq 36.

(127) Ruckenstein, E.; Liu, H. Self-Diffusion in Gases and Liquids. Ind. Eng. Chem. Res. 1997, 36, 3927-3936.

(128) Woodcock, L. V. Equation of State for the Viscosity of Lennard-Jones Fluids. AIChE J. 2006, 52, 438-446. 